\title{
IONIZED GAS IN THE GALACTIC CENTER: NEW OBSERVATIONS AND INTERPRETATION
}

\author{
Wesley T. Irons ${ }^{1}$, John H. LaCY ${ }^{1,3}$, AND Matthew J. RichteR ${ }^{2,3}$ \\ ${ }^{1}$ Department of Astronomy, University of Texas, Austin, TX 78712, USA; lacy@ astro.as.utexas.edu \\ ${ }^{2}$ Department of Physics, University of California, Davis, CA 95616, USA \\ Received 2012 April 5; accepted 2012 June 12; published 2012 July 31
}

\begin{abstract}
We present new observations of the [Ne II] emission from the ionized gas in Sgr A West with improved resolution and sensitivity. About half of the emission comes from gas with kinematics indicating it is orbiting in a plane tipped about $25^{\circ}$ from the Galactic plane. This plane is consistent with that derived previously for the circumnuclear molecular disk and the northern arm and western arc ionized features. However, unlike most previous studies, we conclude that the ionized gas is not moving along the ionized features, but on more nearly circular paths. The observed speeds are close to, but probably somewhat less than expected for orbital motions in the potential of the central black hole and stars and have a small inward component. The spatial distribution of the emission is well fitted by a spiral pattern. We discuss possible physical explanations for the spatial distribution and kinematics of the ionized gas, and conclude that both may be best explained by a one-armed spiral density wave, which also accounts for both the observed low velocities and the inward velocity component. We suggest that a density wave may result from the precession of elliptical orbits in the potential of the black hole and stellar mass distribution.
\end{abstract}

Key words: Galaxy: center - Galaxy: kinematics and dynamics - H II regions - ISM: kinematics and dynamics

Online-only material: Supplemental data (FITS) file (tar.gz)

\section{INTRODUCTION}

The center of the Milky Way has been the subject of intense study since the observation of infrared emission from the central star cluster (Becklin \& Neugebauer 1968) and radio wavelength emission from the ionized gas in Sgr A West (Downes \& Martin 1971) and the central compact object Sgr A* (Balick \& Brown 1974). Being nearly 100 times closer than any other major galactic nucleus, our Galactic center provides the best opportunity to observe the interaction of stars and gas with a supermassive black hole (SMBH). Numerous authors have reviewed the contents and phenomena found in the Galactic center. Morris \& Serabyn (1996), Mezger et al. (1996), and Genzel et al. (2010) discuss observations of the interstellar gas that is most relevant for this paper.

Observations of stellar proper motions and radial velocities (Ghez et al. 2008; Gillessen et al. 2009) give a distance to the Galactic center of $8.3 \mathrm{kpc}$ (corresponding to an image scale of $25^{\prime \prime} \mathrm{pc}^{-1}$ ) and a black hole mass of $4.3 \times 10^{6} M_{\odot}$. Stellar spectra and imaging give evidence for recent star formation or the capture of a recently formed star cluster (Levin \& Beloborodov 2003; Gerhard 2001). The stellar mass distribution in Sgr A West is not well known. If there is an equilibrium stellar cusp, as expected for a cluster around an SMBH (Bahcall \& Wolf 1976), the radial dependence of the stellar density can be described by a broken power law with a slope $\gamma \approx 1.3$ for the cusp and $\gamma \approx 1.8$ outside the cusp (Genzel et al. 2003; Schödel et al. 2007; Genzel et al. 2010). Merritt (2010) describes how the absence of a Bahcall-Wolf cusp is plausible assuming that the mass in the inner parsec is traced by old stars which would indicate a low-density core with radius $\approx 0.5 \mathrm{pc}$. Some recent papers (Buchholz et al. 2009; Do et al. 2009; Bartko et al. 2010) have suggested that there may be a relatively flat stellar density inside of $\sim 1 \mathrm{pc}$.

\footnotetext{
3 Visiting Astronomer at the Infrared Telescope Facility, which is operated by the University of Hawaii under Cooperative Agreement No. NCC 5-538 with the National Aeronautics and Space Administration, Office of Space Science, Planetary Astronomy Program.
}

The most prominent interstellar matter in the central $1.5 \mathrm{pc}$ is the ionized gas and the associated warm dust (Rieke \& Rieke 1988). This gas has the appearance of a clumpy, filamentary multi-armed spiral (Lo \& Claussen 1983; Serabyn \& Lacy 1985). The mass of the ionized gas is several tens of $M_{\odot}$. Neutral atomic gas is also present in the inner few pc (Jackson et al. 1993). Although it is more difficult to observe, its mass is a factor of $\sim 10$ times that of the ionized gas. Beyond $\sim 1.5 \mathrm{pc}$ and extending out to $\sim 10 \mathrm{pc}$, the interstellar gas is mostly molecular, and is referred to as the circumnuclear disk or CND (Becklin et al. 1982; Güsten et al. 1987; Christopher et al. 2005; MonteroCastaño et al. 2009; Oka et al. 2011). Estimates for the mass of the CND range from a few $10^{4} M_{\odot}$, based on millimeter dust emission (Mezger et al. 1989; Davidson et al. 1992; Etxaluze et al. 2011), to $10^{6} M_{\odot}$, based on virial masses of molecular clumps (Christopher et al. 2005; Montero-Castaño et al. 2009).

Observations of infrared and radio hydrogen recombination lines (RRLs; Roberts \& Goss 1993; Herbst et al. 1993; Paumard et al. 2003; Zhao et al. 2009) and infrared fine-structure lines (Wollman et al. 1977; Lacy et al. 1980, 1991; Serabyn et al. 1988) provide information on the motion of the ionized gas through Doppler shifts. The overall pattern is consistent with expectations for orbital motions in a potential dominated by the massive black hole: the highest velocities are found within a few arcseconds of Sgr A*, and velocities tend to decrease going outward. Much of the gas appears to be near a plane tipped $\sim 25^{\circ}$ from the Galactic plane, with redshifts seen toward positive Galactic longitudes and blueshifts generally seen toward negative longitudes. The motions of the molecular gas in the CND are also mostly in the sense of Galactic rotation, but with a roughly flat rotation curve, as distributed mass makes a larger contribution to the gravitational potential farther from the center.

Several models have been proposed to explain the gas kinematics. Lacy et al. (1980) originally saw the gas as being in a number of independently orbiting clouds, but better imaging, especially with the Very Large Array (VLA; Lo \& Claussen 1983), showed that the ionized gas was better described as 


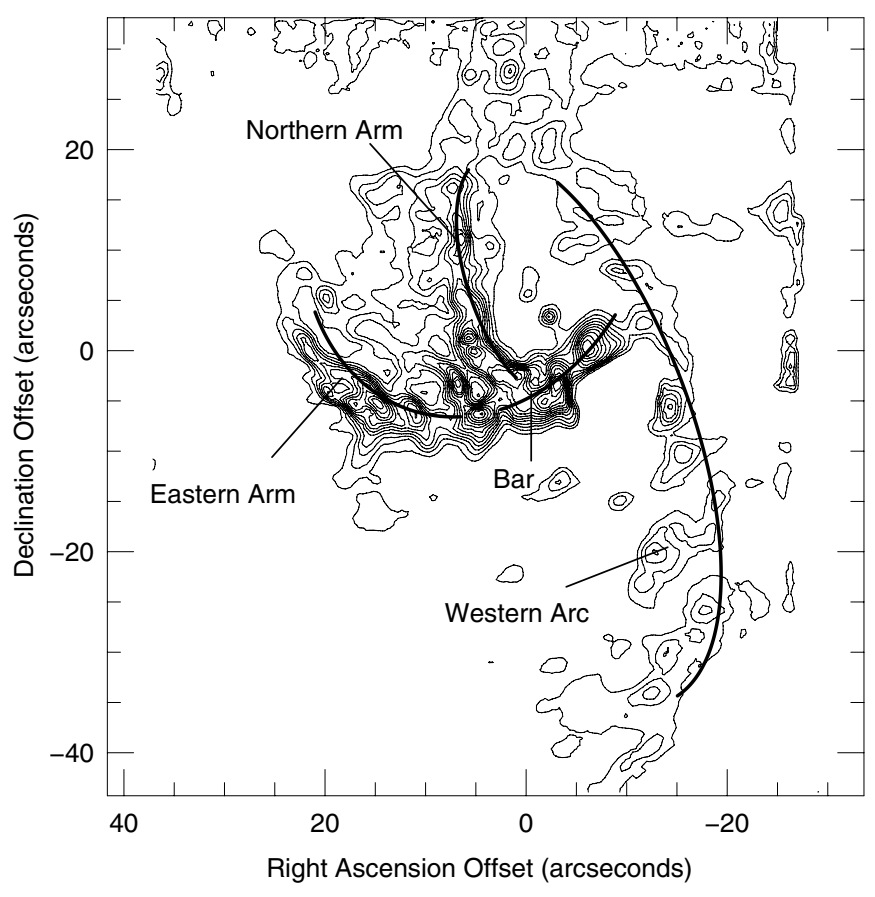

Figure 1. Contour plot of [ $\mathrm{Ne}$ II] emission, with a nonlinear stretch, shown here with the various structures labeled. Offsets are from Sgr $A^{*}$ at $17^{\mathrm{h}} 45^{\mathrm{m}} 40^{\mathrm{s}} .04$ $-29^{\circ} 00^{\prime} 28^{\prime \prime} .11$ (J2000).

(Supplementary data (FITS images) of this figure are available in the online journal.)

a collection of streamers, with the "clouds" being peaks in the emission along the streamers. Serabyn \& Lacy (1985) and Serabyn et al. (1988) found that Doppler shifts vary smoothly along the streamers. They modeled the "western arc" (see Figure 1) as the ionized inner rim of the CND in a nearly circular orbit around the center, and the "northern arm" as a flow of gas approaching the center. Lacy et al. (1991) obtained a complete data cube of the [Ne II] emission from the inner $60^{\prime \prime} \times$ $90^{\prime \prime}$ and concluded that the gas kinematics of the western arc and northern arm were better modeled with circular motions, rather than motions along the streamers. They argued that the western arc and northern arm are orbiting in the same plane as the CND and that they could be joined at their north ends to form a single spiral feature. The main problem with their interpretation was the lack of a physical explanation for the spiral. They suggested that it could be a density wave or a spiraling inflow affected by both gravitational and viscous forces, but in both cases it was hard to identify the forces responsible for organizing the gas into a spiral pattern. Observations of infrared and RRL emission led various authors (Sanders 1998; Vollmer \& Duschl 2000; Liszt 2003; Paumard et al. 2004) to return to the tidally stretched cloud model. Zhao et al. (2009) strengthened this model by including proper motions of the ionized gas streamers. They fitted observations of the western arc, northern arm, and eastern arm with elliptical Keplerian orbits in the potential of the central black hole.

Non-gravitational forces may also influence the gas distribution and motions. Aitken et al. $(1991,1998)$ and Glasse et al. (2003) observed polarized emission from the dust in the northern arm and bar region, indicating that mGauss magnetic fields are aligned along the ionized streamers. Aitken et al. (1998) interpreted variations in the polarization to give a measure of the inclination of the magnetic fields from the plane of the sky. Assuming that the flows are along the field lines, they obtained information about the three-dimensional structure of the gas orbits. Stellar winds apparently also affect the ionized gas. In several cases, bow shocks are seen around stars, presumably as the stars move through the ionized medium or a wind from the central region blows past the stars (Serabyn et al. 1991; Geballe et al. 2004).

We have made new observations of the [Ne II] emission from Sgr A West with improved spectral and spatial resolution, as well as improved sensitivity. In this paper, we present these observations and compare them to the different models of the ionized gas kinematics.

\section{OBSERVATIONS}

[Ne II] line emission from Sgr A West was observed in 2003 June with TEXES on the NASA IRTF. TEXES, the Texas Echelon Cross Echelle Spectrograph (Lacy et al. 2002), is a high-resolution spectrograph for wavelengths of 5-25 $\mu \mathrm{m}$. At the $12.8 \mu \mathrm{m}\left(780.42 \mathrm{~cm}^{-1}\right)$ wavelength of the [Ne II] finestructure line, it has a spectral resolution of $\sim .01 \mathrm{~cm}^{-1}$, or $4 \mathrm{~km} \mathrm{~s}^{-1}$, and a spatial resolution along the entrance slit of $\sim 1^{\prime \prime}$.3. Spectral and spatial sampling is 4 pixels per resolution element. This is a significant improvement over that of the data cube used by Lacy et al. (1991), which had a spectral resolution of $33 \mathrm{~km} \mathrm{~s}^{-1}$ and a spatial resolution of $\sim 2^{\prime \prime}$. For comparison, the observations presented by Zhao et al. (2009) have a spectral resolution of $14 \mathrm{~km} \mathrm{~s}^{-1}$ (with an $\mathrm{H}$ i thermal line width $\sim 20 \mathrm{~km} \mathrm{~s}^{-1}$ ) and a spatial resolution of $\sim 1^{\prime \prime}$.25. To map Sgr A West, the spectrograph slit was oriented N-S and stepped in 0.7 steps to the east, covering $76^{\prime \prime}$. As the slit length is only $10^{\prime \prime}$, multiple scans were made, separated by $5^{\prime \prime}$ in declination, to cover a region of $76^{\prime \prime} \times 78^{\prime \prime}$ centered near Sgr A* At $12.8 \mu \mathrm{m}$, the echelon spectral orders are about $10 \%$ longer than the detector width, leaving gaps between orders, with $\sim 230 \mathrm{~km} \mathrm{~s}^{-1}$ of each $255 \mathrm{~km} \mathrm{~s}^{-1}$ order covered. To fill in these gaps, we made two sets of observations: one with the spectrum offset to center the red side of each echelon order on the detector array and one with the blue side centered. The spectral coverage of the combined data cube is $-1300-800 \mathrm{~km} \mathrm{~s}^{-1}$.

The individual scans were reduced as described by Lacy et al. (2002), and the sky emission was subtracted from each using the median value along the scan as sky for each spectral pixel. We also subtracted fluctuating water vapor emission by subtracting a multiple of a spectrum obtained from the correlation of each spectral pixel with pixels containing strong water lines. The scans were merged to make two large maps with the spectral settings described above. The merging procedure involved cross-correlating contour maps of the scans to determine the appropriate offsets. The two maps were then combined, averaging overlapping spectral pixels, to complete the data cube. Absolute coordinates were obtained by aligning ionized gas peaks with peaks in the VLA continuum maps. Due to uncertainties in the procedure to merge scans into a map, absolute and relative coordinates have uncertainties $\sim 1^{\prime \prime}$.

The data cube was deconvolved using a maximum entropy method deconvolution routine, utilizing an algorithm from Nityananda \& Narayan (1982). This routine is designed to sharpen the image when the signal is high, while smoothing low signal regions. The routine effectively smoothed out the noise while slightly enhancing the resolution in regions where the line is apparent. The contour map of the data cube, summed over Doppler shifts from $-339 \mathrm{~km} \mathrm{~s}^{-1}$ to $+299 \mathrm{~km} \mathrm{~s}^{-1}$, is shown in Figure 1, with the various filaments labeled. 


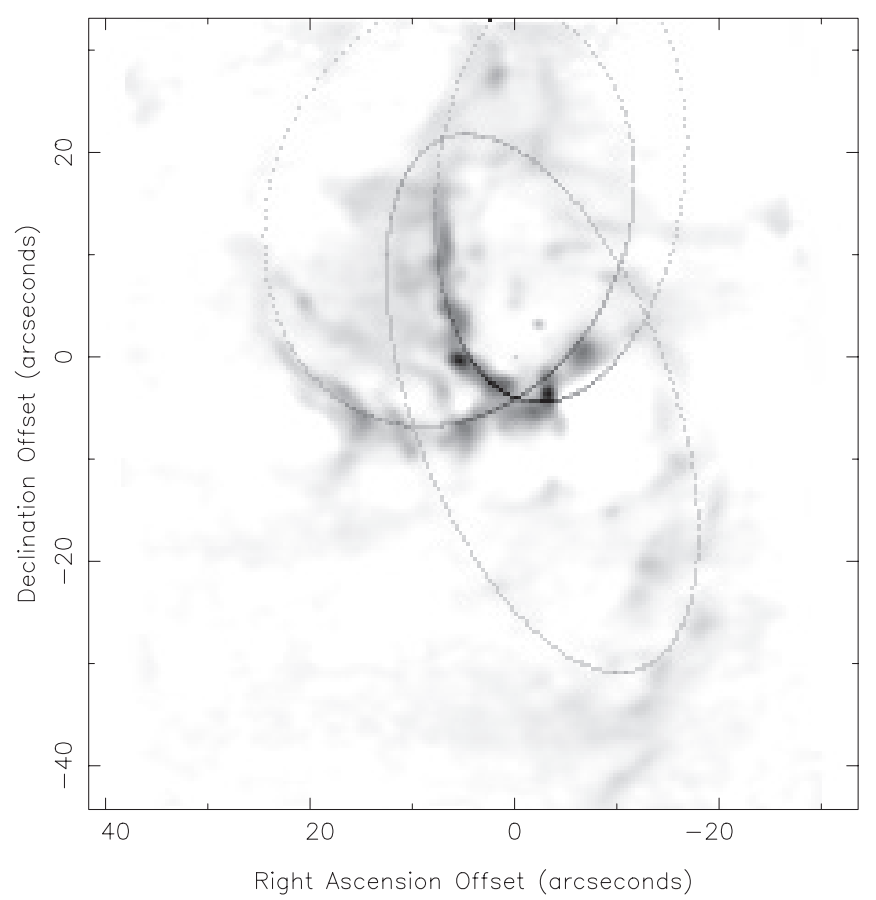

Figure 2. Integrated [Ne II] emission with a nonlinear stretch and the elliptical orbits of Zhao et al. (2009) superposed.

A FITS format deconvolved data cube, spectrally binned by two pixels and including $-670 \mathrm{~km} \mathrm{~s}^{-1}$ to $+345 \mathrm{~km} \mathrm{~s}^{-1}$, is available in the online journal.

\section{KINEMATIC FITTING}

Using the new Galactic center observations of [Ne II] emission, we compare two models for the ionized gas motions in the region. The first models the filaments as tidally stretched clouds in which the gas is flowing along the streamers. For this model, we use the fitting parameters of Zhao et al. (2009) where the gas filaments are taken to be on separate Keplerian orbits. We refer to this as the "ellipse model." This model is the most widely accepted explanation for the ionized gas kinematics. The second model we discuss is similar to the model of Lacy et al. (1991). This model fits the gas on nearly circular orbits around Sgr A* and connects the northern arm and the western arc into a single feature in one plane. We will refer to this model as the "circular orbit model," although we will allow the orbits to have a nonzero radial component. The circular orbit model has not received as much attention, but we are motivated to reevaluate it with the improved data and some discrepancies between the data and the ellipse model.

\subsection{Ellipse Model}

The orbits fitted by Zhao et al. (2009) are shown on the [Ne II] image in Figure 2. To compare our data to this model, we first smoothed the [Ne II] data cube spatially over a square box on the sky of width [17 $R_{\mathrm{pc}}$ pixels], where $R_{\mathrm{pc}}$ is the radial distance from the center in parsecs. The data cube was then tagged with points along the ellipses separated by $1^{\circ}$ in the orbital planes, and spectra were extracted from the tagged points and used to construct the position-velocity $(P-V)$ diagrams shown in Figure 3. The model curves in Figure 3 assume Keplerian motions along the ellipses and an SMBH mass of $4.2 \times 10^{6} M_{\odot}$.
The elliptical orbit models fit rather well spatially, with emission seen typically for $\sim 180^{\circ}$ along each orbit. The spectral fit, however, shows some discrepancies from the data. For the northern arm (Figure 3(a)), the data are systematically offset in velocity from the model. This would be explained if the velocities in the northern arm are not aligned with the streamer as assumed, but are systematically tipped toward circular motions. This anomaly supports the previous assumption by Lacy et al. (1991) that the gas is moving in circular orbits across the filaments. There is a similar anomaly in Figure 3(b), though less pronounced, as the proposed orbit for the western arc is almost circular.

We note that Figure 20 of Zhao et al. (2009) shows a similar discrepancy between the elliptical orbit model and the RRL data. In general, the [ $\mathrm{Ne}$ II] data agree with the RRL data, giving us confidence that the [Ne II] line is a good tracer of the ionized gas.

The kinematic model for the eastern arm (Figure 3(c)) seems to be the least convincing. Unfortunately, the circular orbit model also does not fit the eastern arm kinematics, so it is clear that more work is needed to accurately describe that filament. Liszt (2003) proposes a combined model for the eastern arm and the bar that deserves further study.

The disagreement between the elliptical orbit models and the kinematic data indicates that the gas does not move along the ionized streamers, especially the northern arm. But before discarding this type of model, we should ask whether a tidally stretched cloud is necessarily stretched along its direction of motion. To answer this question, we ran a simple twodimensional hydrodynamic model, similar to that discussed in Section 5.2. In fact, whether the particles in an infalling cloud follow along a narrow streamer depends on the initial conditions of the simulation. In particular, if the cloud initially rotates in the prograde direction, then it forms an arc as it approaches the center, with the particle motions tipped from the arc toward more circular paths. Although this suggests that a tidally stretched cloud model could be made which would agree better with the observations than those we considered, we were not able to find a very acceptable model of this type. Consequently, we now proceed to consider other types of models.

\subsection{Circular Orbit Model}

The discrepancies in the ellipse models provide motivation to reevaluate the circular orbit model of Lacy et al. (1991) with the improved TEXES data. Assuming circular velocities, we fit the [Ne II] emission spectrally with parameters describing the plane in which the gas is moving (that is, an inclination angle, $l$, and the position angle of the line of nodes, $\Omega$ ), along with those describing the mass distribution in the region. The orbital speed was taken to be $v=[G M(r) / r]^{1 / 2}$, appropriate for orbital motions in a spherically symmetric mass distribution. If non-gravitational forces act on the gas or if the mass distribution is not spherically symmetric, $M(r)$ can be taken to be a way of parameterizing the forces acting on the gas. The best fit was that plane which fit the most gas from the entire data cube on circular orbits, using a procedure described below. We also considered models in which we included a correction to the circular Doppler velocity by adding an inward (or outward) radial component that allows the gas to be spiraling into (or out of) the Galactic center. This radial component was made a free parameter and could take the value zero.

For our calculations, we used two different stellar mass distributions. The first is a power-law distribution adopted from 

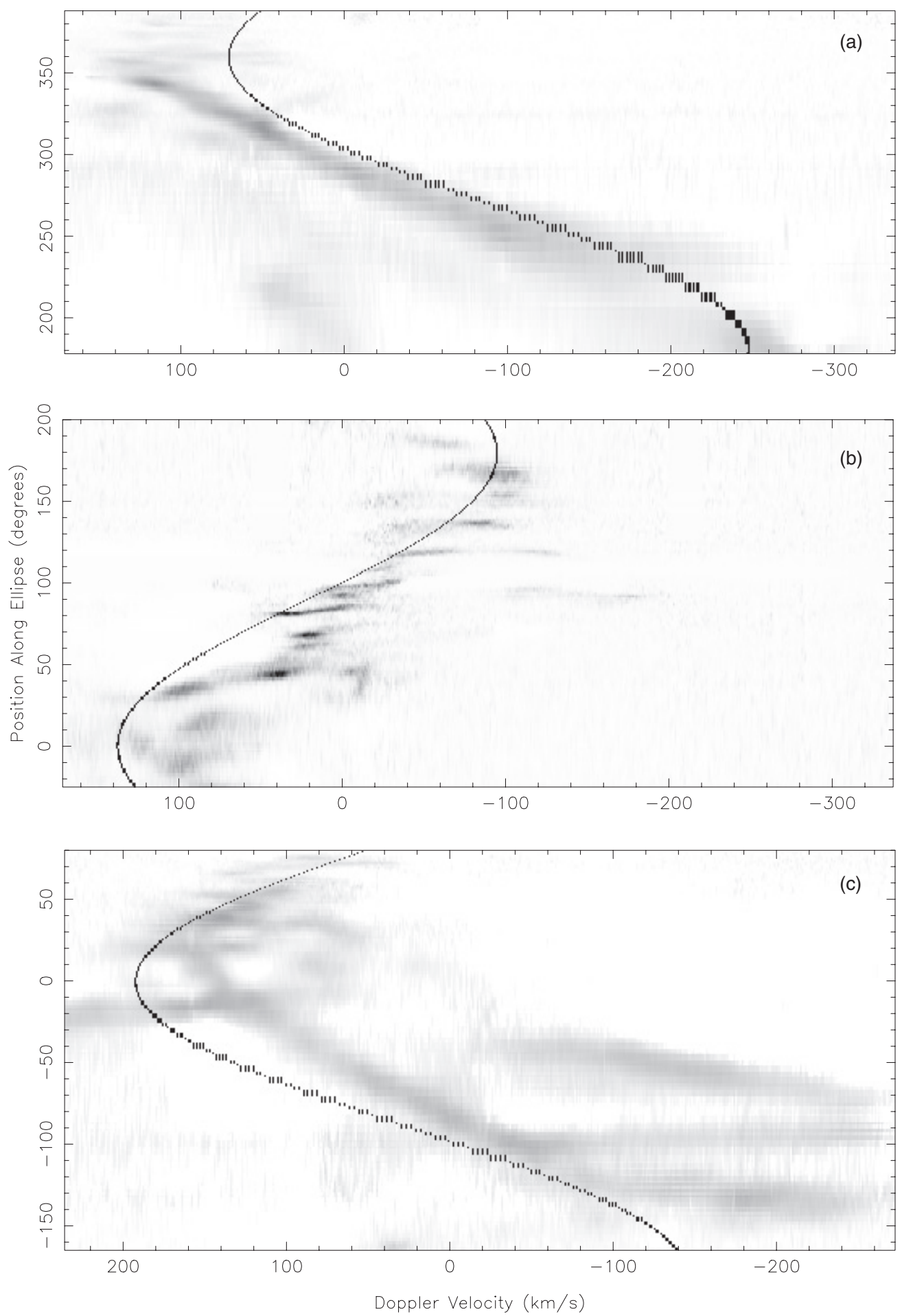

Figure 3. Position-velocity diagrams extracted from the [Ne II] data cube along the ellipses, along with the calculated velocity patterns for the ellipse model. Vertical axes are linear in angle around the ellipses running clockwise along the streamers. Top to bottom: the northern arm, the western arc, and the eastern arm.

Genzel et al. (2010): ${ }^{4}$

$$
M_{*}(r) \approx M_{1}\left(\frac{r}{1.0 \mathrm{pc}}\right)^{\alpha}
$$

\footnotetext{
4 The distribution determined by Genzel et al. (2010) was a broken power law. We are using a slightly simplified form of their mass distribution for $r>0.25 \mathrm{pc}$, noting that our observations are not particularly sensitive to the stellar mass and that these numbers contain large systematic uncertainties.
}

Here, we did not allow $M_{1}$ and the power $\alpha$ to be free parameters as the ionized gas is not especially sensitive to the stellar mass distribution, so they will take the values $1.0 \times 10^{6} M_{\odot}$ and 1.2, respectively. The second mass distribution considered assumes a Lorentzian density distribution:

$$
\rho(r) \approx \rho_{\circ}\left(\frac{R_{c}^{2}}{r^{2}+R_{c}^{2}}\right),
$$


where $\rho_{\circ}$ is the density at the center and $R_{c}$ is the core radius. This distribution flattens out within the core radius and drops as $1 / r^{2}$ outside the core radius, roughly approximating an isothermal star cluster. Equation (2) can be integrated to arrive at a mass distribution:

$$
M_{*}(r) \approx 4 \pi \rho_{\circ} R_{c}^{2}\left(r-R_{c} \arctan \left(\frac{r}{R_{c}}\right)\right) .
$$

For each mass distribution model, we determined the best fit allowing the dynamical mass of $\mathrm{Sgr} \mathrm{A}^{*}$ and the core stellar density, as well as the disk plane orientation, to be free parameters. Because the black hole mass is well known from stellar observations (Ghez et al. 2008; Gillessen et al. 2009) and the $[\mathrm{Ne} \mathrm{II}]$ data are not especially sensitive to the stellar mass, it was useful to also find the best fits holding these parameters constant $\left(M_{\bullet}=4.2 \times 10^{6} M_{\odot}, \rho_{\circ}=1 \times 10^{6} M_{\odot} \mathrm{pc}^{-3}, R_{c}=\right.$ $0.5 \mathrm{pc})$.

To determine the best parameters for the orbiting gas, we first searched through parameter space to find the best-fit orbital plane. For each set of parameters describing a plane and orbital motion, we calculated the Doppler velocity at each spatial point in the data cube. We then shifted the spectrum at each point by that velocity so that all the emission fitting circular velocities in that plane would be aligned at the zero velocity wavelength, and collapsed the map into a single spectrum. We then compared how well the emission in the data cube fit the model velocities in a given plane with any other plane. To illustrate what this routine does, we show in Figure 4 the collapsed spectrum before the spectral shifting (panel (a)) and the spectrum corresponding to a good fit (panel (b)). Of course, this method will always provide a "best-fit" set of parameters for any velocity model, and it is necessary to determine how good such a fit is. We have no quantitative way of stating error bars for the fit parameters because some velocity variations are expected due to turbulence, and the discrepancies from the fit are probably not due to random noise with any known distribution. In addition, we do not expect our model to fit all of the emission, as we know that not all of the gas lies in a single plane. After smoothing the shifted spectrum with a Gaussian, we can determine the flux at zero Doppler velocity, and compare the results with each set of parameters. Alternatively, we can compare the flux in an interval around zero Doppler velocity. The fitting procedure utilized the first method with a smoothing Gaussian defined by $\exp \left[-\Delta p^{2} / \sigma^{2}\right]$, where $\Delta p$ is the separation from the spectral pixel to the zero velocity pixel and $\sigma=5 \mathrm{~km} \mathrm{~s}^{-1}$. We also give the results using the second method using a $\pm 30 \mathrm{~km} \mathrm{~s}^{-1}$ interval in Table 1 . For a good fit, we would expect to gather a higher percentage of the total $[\mathrm{Ne}$ II] emission in a lower percentage of the total velocity range. We provide these ratios as an easy way to interpret the results of the fitting, though this can be done essentially by looking at the Gaussian fit to the collapsed spectrum that results from this routine.

In order to allow the gas to spiral into or out of the Galactic center, we added to the calculated circular velocities a radial component which is equal to the fraction $a / r$ of the angular component, where $a$ is a free parameter. The gas can then be viewed as flowing along a linear spiral, $r_{\text {flow }}(\theta)=a \theta$, where $\theta$ is measured in radians. The collapsed spectrum in Figure 4(b) was made using the mass distribution in Equation (3) and the parameters, $l=66^{\circ}, \Omega=23^{\circ}, M_{\bullet}=3.5 \times 10^{6} M_{\odot}$, $\rho_{\circ}=2.5 \times 10^{5} M_{\odot} \mathrm{pc}^{-3}, R_{c}=0.5 \mathrm{pc}$, and $a=-0.06 \mathrm{pc}$.

The results of this fitting are compiled in Table 1. It includes the best fits for both stellar mass distributions, Equations (1)
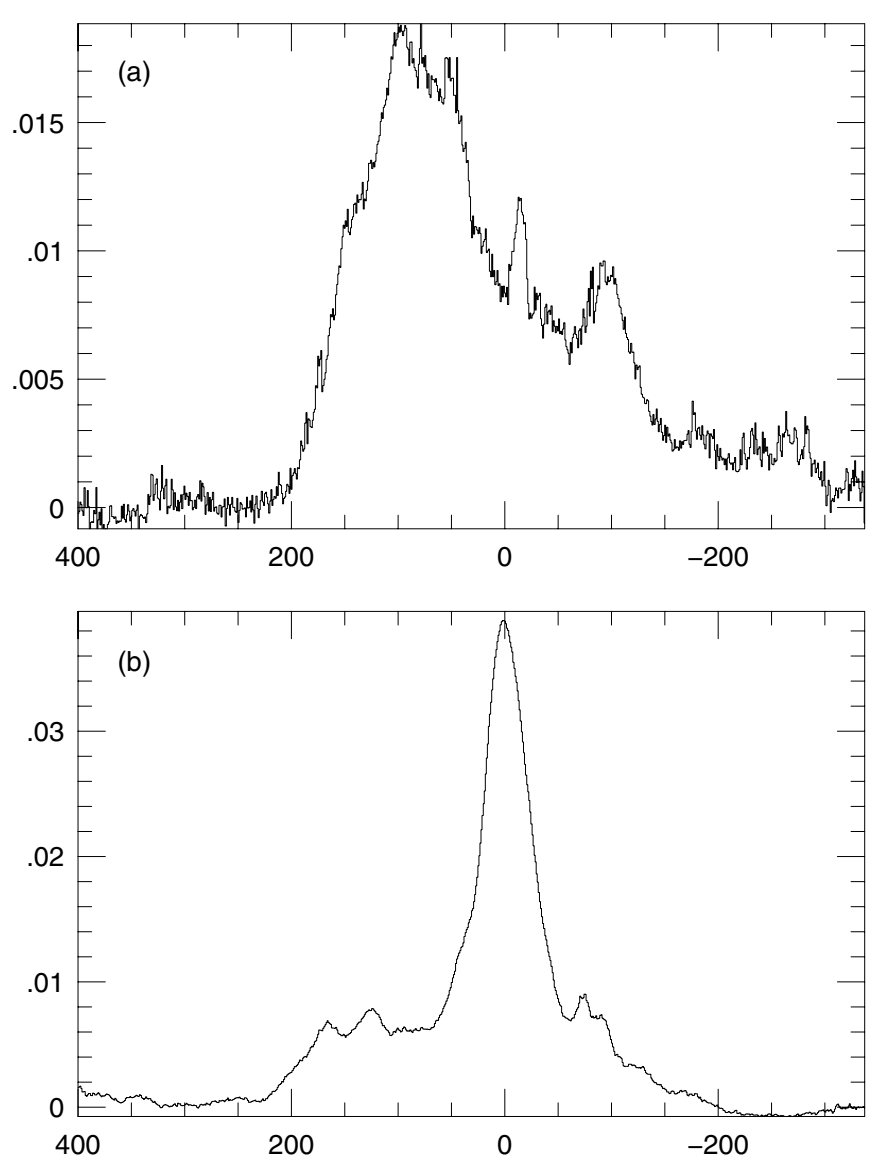

Figure 4. (a) Spectrum taken by collapsing the data cube spatially before shifting. Here, $15 \%$ of the [Ne II] emission fits within $10 \%$ of the velocity range centered at zero Doppler velocity. (b) Spectrum after shifting the data cube to fit circular motion. Now, $45 \%$ of the [Ne II] emission fits within $10 \%$ of the velocity range centered at zero Doppler velocity. This is the "goodness-of-fit" parameter for the routine. The horizontal axis is Doppler velocity $\left(\mathrm{km} \mathrm{s}^{-1}\right)$. The smoother appearance of panel (b) is a result of shifting and averaging systematic noise from the background subtraction.

and (3), holding the black hole mass fixed and allowing it to change. We also determined the best fit using $\mathrm{HCN}(4-3)$ data from Montero-Castaño et al. (2009), to determine whether the orbital plane and mass distribution that fit the ionized gas also fit the molecular gas in the CND. We also quote a "goodness parameter" which is just the ratio of the emission that fits within $\pm 30 \mathrm{~km} \mathrm{~s}^{-1}$ to the total emission in the map. Again, this is not the same as quoting error bars, but it provides a rough measure of whether this fit could have been achieved by accident.

To accompany Table 1 and the "goodness parameter," Figure 5 shows the collapsed, shifted spectra corresponding to the different fits in the table. Each set of conditions in Table 1 provides an acceptable best fit, as seen in Figure 5. The ionized gas is not a good measure of the shape of the mass distribution and whether the mass should be in the black hole or in the stars. With the different mass distributions and mass constraints, the best fits all give roughly the same orbital plane, with the exception of row 8 , which is described below.

Note that when using Equation (3) for the stellar mass distribution, the best-fit black hole mass is smaller than the accepted values from the literature. Alternatively, if we constrain the mass to be $4.2 \times 10^{6} M_{\odot}$, then we prefer no stars in the region. Although there clearly are stars in Sgr A West, this result may be consistent with the suggestion that the stellar density is flat 
Table 1

Table of Parameters

\begin{tabular}{|c|c|c|c|c|c|c|c|}
\hline Stellar Distribution & $M_{\bullet}\left(10^{6} M_{\odot}\right)$ & $\begin{array}{c}\rho_{\circ}\left(10^{6} M_{\odot} \mathrm{pc}^{-3}\right) \\
\text { or } M_{1}\left(10^{6} M_{\odot}\right)\end{array}$ & $\begin{array}{c}R_{c}(\mathrm{pc}) \\
\quad \text { or } \alpha\end{array}$ & 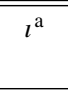 & $\Omega^{\mathrm{b}}$ & $\begin{array}{c}a^{\mathrm{c}} \\
(\mathrm{pc})\end{array}$ & Goodness Parameter $^{\mathrm{d}}$ \\
\hline (1) Equation (1) $\left(M_{\bullet}\right.$ fixed) & 4.2 & 1 & 1.2 & $70^{\circ}$ & $21^{\circ}$ & -0.05 & 0.41 \\
\hline (2) Equation (1) $\left(M_{\bullet}\right.$ free $)$ & 2.6 & 1 & 1.2 & $63^{\circ}$ & $24^{\circ}$ & -0.07 & 0.45 \\
\hline (3) Equation (1) (HCN data, $M_{\bullet}$ fixed) & 4.2 & 1 & 1.2 & $78^{\circ}$ & $29^{\circ}$ & -0.42 & 0.60 \\
\hline (4) Equation (3) ( $M_{\bullet}$ fixed) & 4.2 & $0^{\mathrm{e}}$ & 0 & $68^{\circ}$ & $23^{\circ}$ & -0.06 & 0.43 \\
\hline (5) Equation (3) $\left(M_{\bullet}\right.$ free) & 3.5 & 0.25 & 0.5 & $66^{\circ}$ & $23^{\circ}$ & -0.06 & 0.45 \\
\hline (6) Equation (3) (All Mass Fixed) & 4.2 & 1.0 & 0.5 & $72^{\circ}$ & $21^{\circ}$ & -0.04 & 0.39 \\
\hline (7) Equation (3) (HCN data, $M_{\bullet}$ fixed) & 4.2 & 0.25 & 0.75 & $77^{\circ}$ & $28^{\circ}$ & -0.38 & 0.61 \\
\hline (8) Equation (3) (a fixed) $)^{f}$ & 1.4 & 0.25 & 2.75 & $66^{\circ}$ & $42^{\circ}$ & -0.27 & 0.38 \\
\hline
\end{tabular}

Notes. Best-fit parameters for circular motion in a plane (with a small radial correction, $a$ ) for [Ne II] emission and HCN(4-3) emission in the Galactic center for different stellar mass distributions holding the SMBH mass fixed at $4.2 \times 10^{6} M_{\odot}$ and allowing it to be a free parameter.

${ }^{a}$ The inclination is the angle between the sky plane and the plane of the disk.

${ }^{b}$ The angle of line of nodes on the sky is the angle between north and the intersection of the disk plane and the sky plane.

${ }^{c}$ The radial velocity parameter $(a)$ is the outward velocity component as a fraction of the angular velocity (that is, the gravitational circular velocity) multiplied by radius from the center in parsecs. So at a distance of $1 \mathrm{pc}$, and an angular velocity of $100 \mathrm{~km} \mathrm{~s}^{-1}, a=-0.05 \mathrm{pc}$ implies and inward radial velocity of $5 \mathrm{~km} \mathrm{~s}^{-1}$.

d This is our "goodness-of-fit" measurement (see Figure 5). Note that for the unshifted spectrum this ratio is 0.15 .

${ }^{\mathrm{e}}$ Consistent with the kinematics suggesting a black hole mass that is too small, if we force the black hole to be $4.2 \times 10^{6} M_{\odot}$, then we prefer no stars. Naturally, we do not purport that there are no stars, nor that the black hole mass is less than $4 \times 10^{6} M_{\odot}$, however it is interesting to note what might cause these anomalies in the observational kinematics. We speculate in Section 5.

${ }^{\mathrm{f}}$ After fitting a physical spiral with the parameters from row 5 in this table, we fixed the inward velocity component to fit gas flowing along this spiral to check that this parameter is not redundant with the angle of line of nodes. The best fit constraining $a=-0.27 \mathrm{pc}$ did change $\Omega$ considerably, but is a much worse fit kinematically.

within $\sim 1 \mathrm{pc}$, with a possible hole in the inner few arcseconds (Buchholz et al. 2009; Do et al. 2009). We will speculate further on the low mass results in Section 5, but for now we will just examine the results shown in the fifth row of Table 1.

In Figure 6(a), we show the spatial distribution of the emission within $\pm 30 \mathrm{~km} \mathrm{~s}^{-1}$ of the velocity of the best-fitting disk model. This emission gives a strong impression of a spiral pattern, as noted by Lacy et al. (1991), and (partly to lead the eye) we superimpose on the image a nearly linear (Archimedean) spiral in the disk plane given by $r(\theta)=0.27 \mathrm{pc} \times \theta(\mathrm{rad})^{0.93}$. Note that the disk plane used is that derived from the gas kinematics, not the spatial pattern, but it allows a good fit to the pattern. To define the spatial distribution for the spiral, we must specify another parameter which we take as the third Euler angle, $\phi$ (the others being $\imath$ and $\Omega$ ). The angle $\phi$ describes the starting point of the spiral. Its effect is the same as adding a constant to $r(\theta)$ and letting $\theta$ go negative. In Figure $6(\mathrm{a}), \phi=274^{\circ}$. We note that our kinematic model involves gas moving along almost circular orbits, not along the spiral, implying that gas moves across the streamers. For the kinematic model presented here, we use the parameters from the fifth row of Table 1. The disk parameters for the spiral structure are similar to those used by Lacy et al. (1991) and by Zhao et al. (2009) for the western arc, with an inclination angle $l=66^{\circ}$ and an angle of line of nodes $\Omega=23^{\circ}$. We display in Figure 6(b) the integrated [Ne II] emission with the emission shown in Figure 6(a) masked out to see the gas that does not lie in the fitted plane. Comparing to Figure 1, we can see from Figures 6(a) and (b) that most of the northern arm and western arc emission fits the velocity pattern described above, while the eastern arm and bar as well as diffuse emission do not. Note that the integrated intensity in Figure 6(a) is $45 \%$ of that for the whole map (Figure 1). This is the basis of the "goodness of fit" for our method. It strengthens the argument that the fitting routine is not merely collecting random gas coincidentally if the percentage of the total emission that fits within a certain velocity range is greater than the percentage of the total velocity range in which it fits. So, in this case, the $\pm 30 \mathrm{~km} \mathrm{~s}^{-1}$ range is less than $10 \%$ of the total velocity range for the map, while the emission collected in this range is about $45 \%$ of the total emission.

To determine how well the velocities of the gas match circular orbits as opposed to motions along the features, we compare the $P-V$ diagrams for gas along the spiral shown in Figure 6(a), using the velocity patterns for purely circular orbits (row 5 in Table 1 , but with $a=0 \mathrm{pc}$ ) and for motion along the spiral (row 5 in Table 1, but with $a=-0.27 \mathrm{pc}$ ). These two models are superposed on the $P-V$ diagram in Figure 7(a). The best-fit parameters include a small inward radial component to the velocity ( $a=-0.06 \mathrm{pc}$ ), which is included in the $P-V$ diagram in Figure 7(b). For the purely circular velocity pattern (lower curve in Figure 7(a)), there is a slight offset in position angle along the northern arm, which validates the small nonzero inward radial velocity component in the best fit. The best-fit parameters, including the radial velocity correction, make an excellent fit to the data (Figure 7(b)). We also tried to apply an inward radial velocity component with a constant pitch angle, so the radial component would be $c v_{\theta}$ rather than $(a / r) v_{\theta}$. Here, the parameter $c$ just shifts the $P-V$ diagram up or down depending on the sign. The best fit in this case had a much smaller pitch angle, which had a negligible effect on the $P-V$ diagram. This is because when the model is shifted up to agree with the data along the northern arm, the model along the western arc no longer fits. Therefore, the spiraling motion described by $v_{r}=(a / r) v_{\theta}$ fits the data best. The top curve in Figure 7(a) is for motion along the physical spiral in the best-fit plane. We note that the model is offset from the data in the same way the ellipse model was offset. This is further confirmation that the gas is not flowing along the streamers.

Although the best kinematic fit to gas near the northern arm/western arc plane involved little inward motion, we wanted to determine whether motion along a spiral in a different plane could fit the kinematic data while providing an acceptable fit to the spatial distribution. There was not a good fit that worked both 

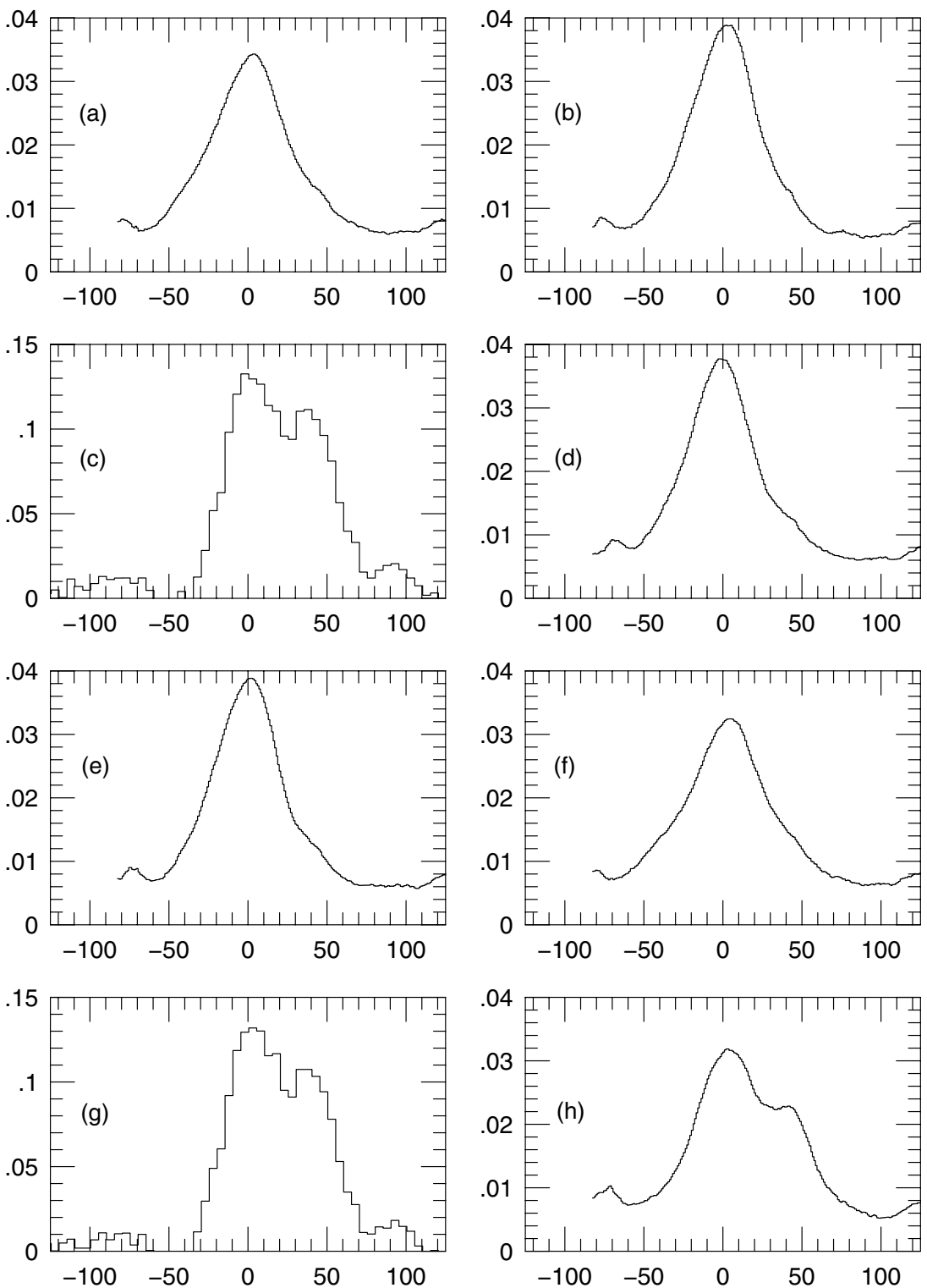

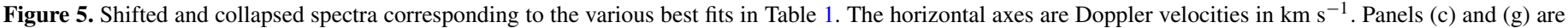
for the Montero-Castaño et al. (2009) HCN data.

spatially and spectrally. The best fit spectrally (for motion along a spiral) corresponded to a very poor fit spatially. By allowing the plane orientation parameter to vary substantially, we were able to fit the kinematics with a larger value of $a(-0.1 \mathrm{pc})$, but the spiral described by this value of $a$ does not follow the observed spatial distribution.

\subsection{Molecular Emission in the Circumnuclear Disk}

We show in Figure 8 a contour map of HCN(4-3) emission from data by Montero-Castaño et al. (2009) superposed on the gray-scale image of the [Ne II] emission. We used a procedure like that used with the [Ne II] data to find the best-fitting plane and mass distribution to explain the HCN kinematics. Much of the gas in the CND has been found to fit in a plane with inclination, $l \approx 70^{\circ}$ (Jackson et al. 1993). We can see from rows 3 and 7 of Table 1 that our results are similar and that this plane is near the plane that fits the [Ne II] kinematics. Note that the CND data are relatively insensitive to the inclination parameter as the rotation curve is mostly flat. When fitting the HCN(4-3) emission, we constrained the black hole mass, as the gas motions in the CND are more sensitive to the stellar mass. The resulting stellar mass parameters are consistent with those derived in row 5 of Table 1 . However, the inward radial velocity component, $a$, is consistent with motions along the spiral that fits the ionized gas observations. At $R=2 \mathrm{pc}$, in the CND, $a=-0.4$ pc corresponds to a pitch angle of $a / R=0.2$ and an inward velocity of $20 \mathrm{~km} \mathrm{~s}^{-1}$.

The western arc has been described as the ionized inner rim of the CND, so it is interesting to ask if the spiral pattern continues into the molecular gas. The $\mathrm{HCN}(4-3)$ emission that fits circular velocities in the plane of the spiral within $\pm 30 \mathrm{~km} \mathrm{~s}^{-1}$ is shown in Figure 9. Included on the HCN image is the spiral from the [Ne II] map in Figure 6(a) (tagged every $1^{\circ}$ ), and the same spiral offset in starting position by $75^{\circ}$ (tagged every $3^{\circ}$ ). Much of the $\mathrm{HCN}(4-3)$ emission lies just outside the spiral used for the ionized gas, or along the second spiral. Figure 10 shows the $\mathrm{HCN} P-V$ diagram extracted from along the outside spiral from Figure 9.

The model in Figure 10 does not fit as well as we had hoped, perhaps because the random motions in the CND are a larger 

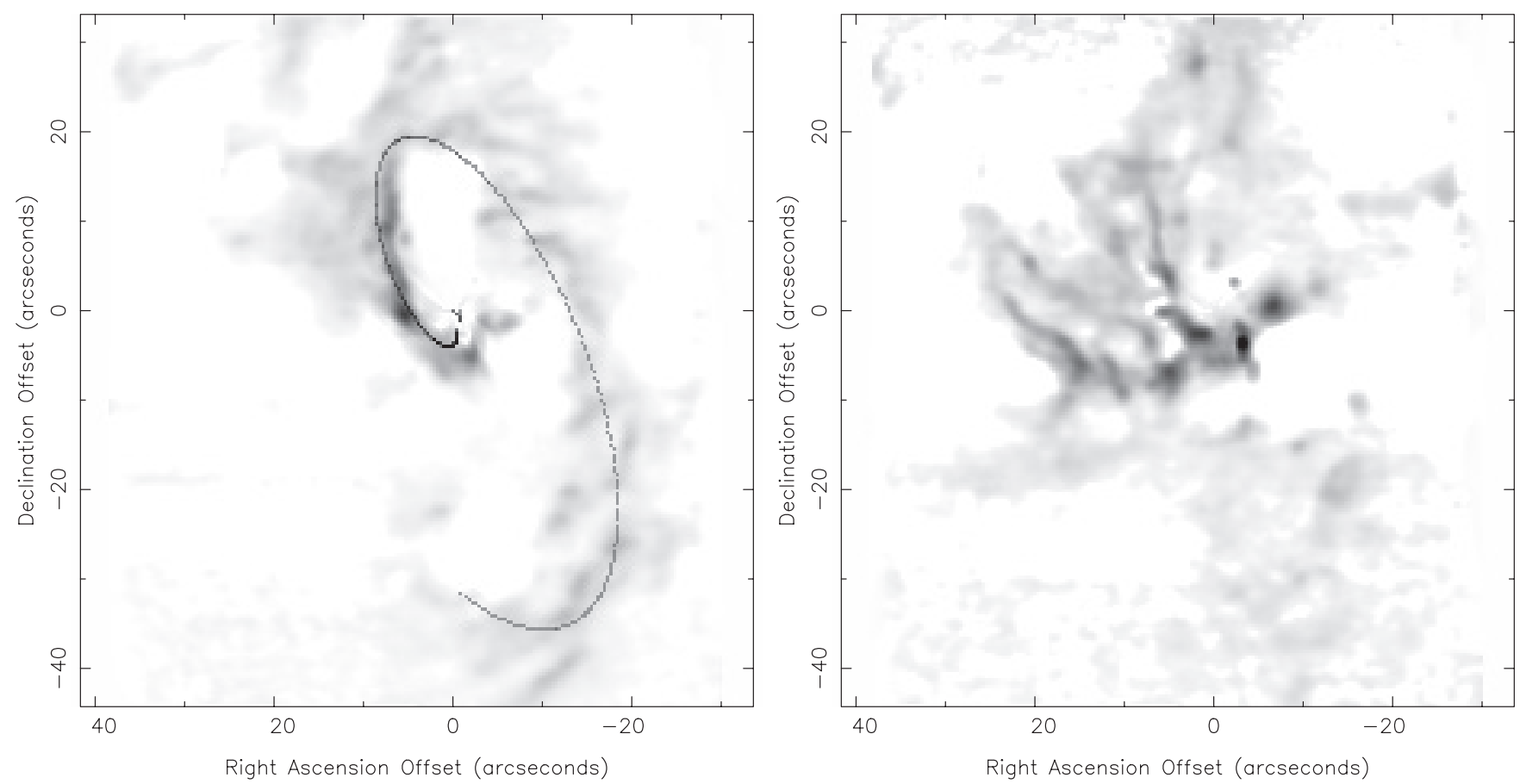

Figure 6. Left: gray-scale image of the ionized gas that fits a nearly circular orbit model in the plane defined by $\imath=66^{\circ}, \Omega=23^{\circ}$, with $a=-0.06$ pc, within $\pm 30 \mathrm{~km} \mathrm{~s}^{-1}$. The spiral model is superposed. Right: the emission in the [Ne II] data cube that does not fit the circular orbit model. That is, emission within $\pm 30 \mathrm{~km} \mathrm{~s}^{-1}$ has been masked out. Both figures are shown with a square root stretch.

fraction of the orbital speed than those in the ionized spiral, though it does fit acceptably well, particularly in the region southwest of Sgr A*.

\section{CONCLUSIONS FROM THE OBSERVATIONS}

Before discussing theoretical models and implications from our observations, we state the conclusions we have drawn that are independent of those models.

Approximately half of the ionic line emission from Sgr A West comes from gas orbiting in a plane tipped about $25^{\circ}$ from the Galactic plane. This plane is coincident within uncertainties with that of the molecular CND.

The gas in the disk plane moves on nearly circular orbits, with only a small inward velocity component. The Doppler pattern is not consistent with motion along the northern arm ionized streamer.

The observed speeds are close to, but probably somewhat less than expected for orbital motions in the gravitational potential of the central SMBH and the distributed mass, as derived from the orbital motions of stars near the black hole and the distribution of stars.

The spatial distribution of the ionized gas in the western arc and northern arm could be described by two ellipses in a plane close to that derived from the [Ne II] line kinematics, but is somewhat better fitted with a single, approximately linear (Archimedean) spiral.

\section{DISCUSSION AND INTERPRETATION}

The fact that the Doppler shifts of the ionized gas in the northern arm and western arc require nearly circular motions rather than motions along the streamers has been pointed out before (Lacy et al. 1991). The elliptical model has become prevalent, perhaps mostly because the interpretation of these features as tidally stretched clouds is so natural, and because it is hard to understand how the ionized gas could be concentrated in an eccentric streamer like the northern arm if the gas motions are circular. We now add to this puzzle about the direction of motion of the gas the observation that the gas speed is probably less than expected in the potential of the black hole and star cluster. Both of these observations need to be explained.

\subsection{Non-gravitational Forces}

We first ask about the possible significance of the fact that the best fit to the kinematic data involves orbital motions somewhat less than the expected Keplerian velocities. One explanation for sub-Keplerian velocities is that there are non-gravitational outward forces on the ionized gas, which might be caused by radiation pressure or by ram pressure of a hot wind. These forces might also contribute to the organization of the gas into the observed spiral pattern.

The radiation pressure due to Thomson scattering of photons from the central star cluster off of electrons can easily be shown to be negligible, as the electron scattering opacity is very small. The dust opacity is larger, so radiation pressure on dust mixed with the ionized gas should be considered. The importance of radiation pressure can be estimated by comparing the momentum flux from a stellar luminosity of a few $10^{6} L_{\odot}$ to the gravitational force on a parcel of gas with a column density corresponding to an optical depth to starlight of the order of one, assuming a normal interstellar dust to gas ratio. This calculation indicates that radiation pressure is a factor $\sim 100$ less important than gravity, so probably not enough to account for the subKeplerian velocities.

If we assume that the x-ray emitting hot gas in the region generates a ram pressure equal to the thermal pressure of the gas, i.e., that the gas moves at the sound speed, then we can estimate the ratio of the outward force from the hot gas to the gravitational force. Using reasonable estimates for the size of a 

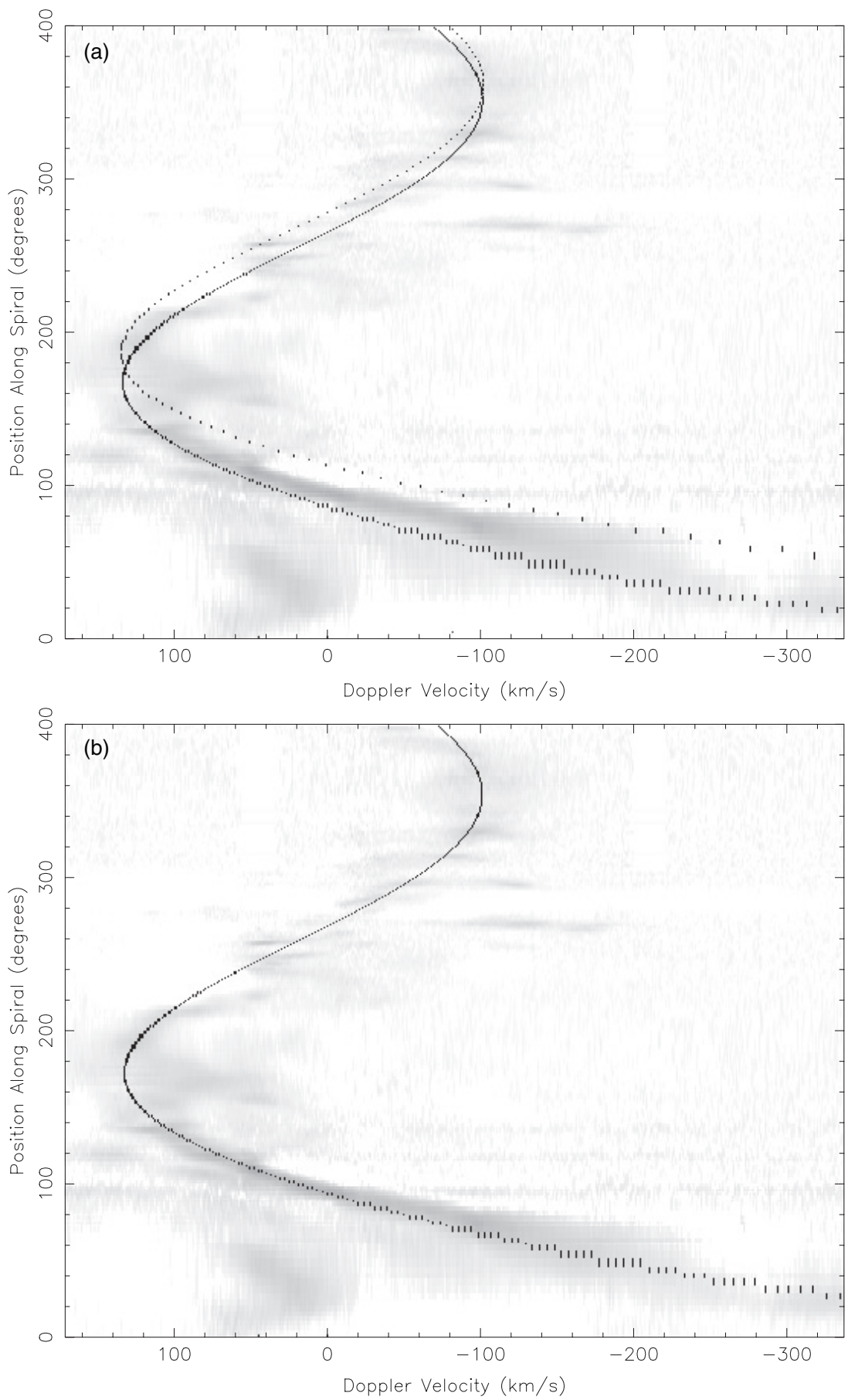

Figure 7. (a) Position-velocity diagram extracted from the [Ne II] data cube along the spiral with calculated velocity patterns for purely circular motion (using parameters from row 5 in Table 1 but with $a=0 \mathrm{pc}$, tagged every $1^{\circ}$ ), and for motion along the spiral (using parameters from row 5 in Table 1 but with $a=-0.27 \mathrm{pc}$, tagged every $3^{\circ}$ ) superposed. (b) Position-velocity diagram extracted from the [Ne II] data cube along the spiral with calculated velocity patterns for the best fit (using parameters from row 5 in Table 1).

gas cloud and the hot gas temperature and electron density from Baganoff et al. (2003), that ratio is about $10^{-3}$ to $10^{-2}$. As an outward force, this will not cause the low velocities observed, but if it is seen as a drag force as the ionized gas flows past the hot gas, then it might be enough to cause the slight spiraling inward motion observed in the ionized gas.

Rather than explaining the possible sub-Keplerian velocities with an outward force, we could explain them by hypothesizing that the gas slows its orbital motion due to a shock as it enters the spiral, and possibly the dissipation of kinetic energy is at least partially responsible for the ionization of the gas. A $20 \%$ decrease in the gas speed would be consistent with the observed Doppler shifts and would correspond to a $40 \%$ loss in kinetic energy. However, even if all of the kinetic energy of the gas entering the northern arm were converted to ionization, the ionization rate would not balance the recombination rate derived from the free-free observation. So although a shock might contribute to the ionization of the gas, and it may affect the orbital speed of the gas, it is not likely to dominate the ionization. 


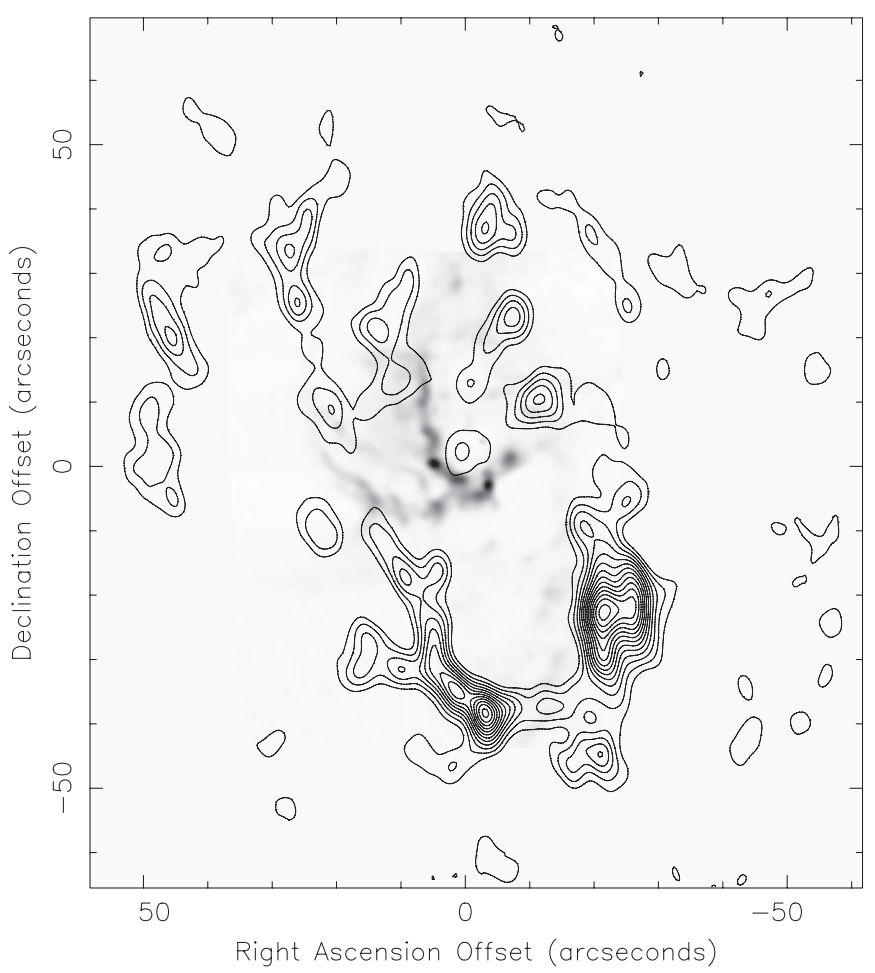

Figure 8. Gray-scale image of [Ne II] emission summed over Doppler shifts from $-339 \mathrm{~km} \mathrm{~s}^{-1}$ to $+299 \mathrm{~km} \mathrm{~s}^{-1}$ using a nonlinear stretch, superposed on a contour map of $\mathrm{HCN}(4-3)$ emission from Montero-Castaño et al. (2009) summed from $-90 \mathrm{~km} \mathrm{~s}^{-1}$ to $+130 \mathrm{~km} \mathrm{~s}^{-1}$.

Magnetic forces are likely to be greater than other nongravitational forces. We can estimate their importance by comparing the magnetic energy density, or pressure, with the kinetic energy density of the orbiting gas and the gas pressure. Aitken et al. (1998) give a lower limit on the field strength of $2 \mathrm{mG}$. This corresponds to an energy density of $B^{2} / 8 \pi=1.6 \times 10^{-7} \mathrm{erg} \mathrm{cm}^{-3}$. If we estimate the ionized gas density to be $10^{4} \mathrm{~cm}^{-3}$ (based on our unpublished observations of [S III], which has a critical density of $1.7 \times 10^{4}$ ), a mean particle mass of $10^{-24} \mathrm{~g}$, an orbital speed of $100 \mathrm{~km} \mathrm{~s}^{-1}$, and a temperature of $8000 \mathrm{~K}$, we calculate a kinetic energy density of $5 \times 10^{-6} \mathrm{erg} \mathrm{cm}^{-3}$ and a gas pressure of $8 \times 10^{-9} \mathrm{erg} \mathrm{cm}^{-3}$. Apparently, the magnetic pressure is substantially greater than the gas pressure, but probably substantially smaller than the kinetic energy density. Consequently, we would expect the field to be carried along by the gas without altering the orbital speeds significantly, although it may exert forces which could perturb the gas motions, possibly influencing the density wave discussed below.

\subsection{A Spiral Density Wave}

We now turn to the origin of the spiral pattern and the observation that the gas moves across the northern arm and western arc streamers. There are two main problems with the idea that the gas motions do not align with the streamers. First, if the streamers move with the gas, then they would wrap up quickly. The orbital period varies from a few $10^{3}$ years at the inner end of the northern arm, $\sim 0.1 \mathrm{pc}$ from the center, to $\sim 10^{5} \mathrm{yr}$ at the inner edge of the CND, so that a wrap should be added to the spiral pattern every few $10^{3} \mathrm{yr}$. On the other hand, if the gas moves across the streamers we need to explain why the emission is concentrated there. We also should explain how ionized gas could move across magnetic field lines, which the

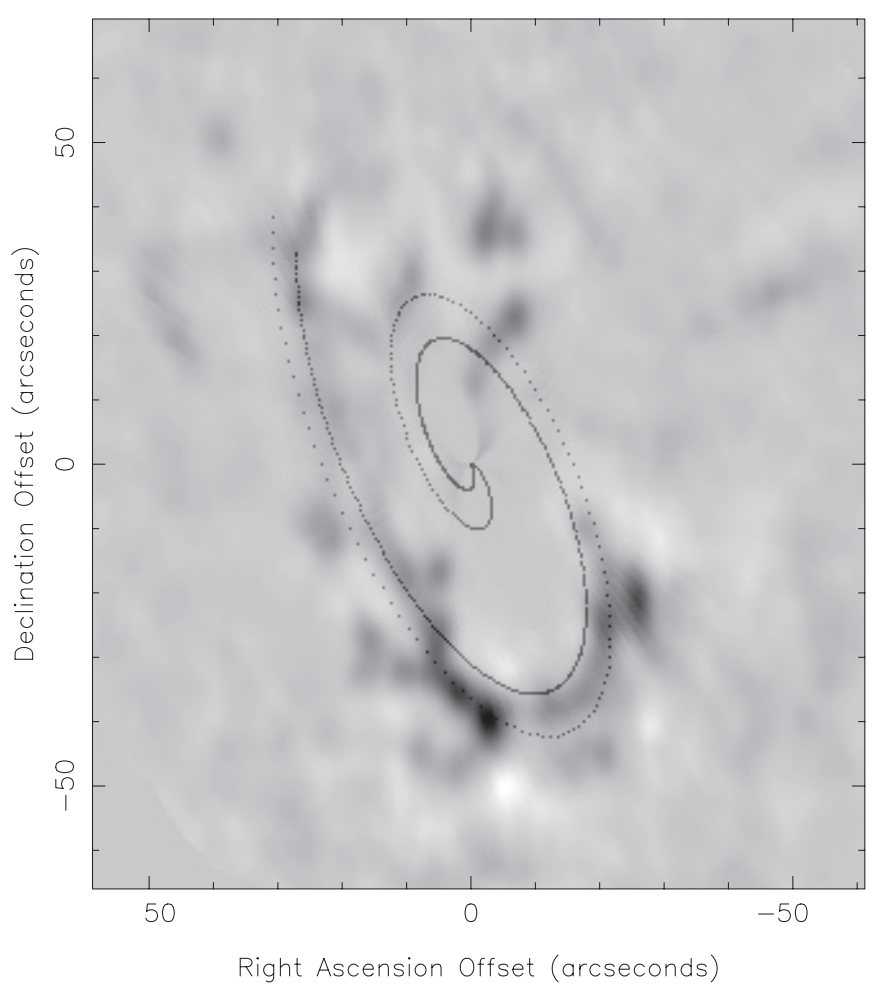

Figure 9. HCN(4-3) emission map showing only the emission that fits circular velocities within $\pm 30 \mathrm{~km} \mathrm{~s}^{-1}$ in the plane described by $\imath=66^{\circ}$ and $\Omega=23^{\circ}$, with the original spiral $\left(1^{\circ}\right.$ steps, $\left.\phi=274^{\circ}\right)$ and a slightly larger spiral $\left(3^{\circ}\right.$ steps, $\left.\phi=199^{\circ}\right)$ superposed.

observations of Aitken et al. (1998) clearly show to be aligned with the northern arm.

In the case of galactic spiral arms, the wrapping problem is normally resolved by assuming that the gas and stars move through the arms, and are observed to be concentrated there because their orbits crowd and spend more time in the arms. Perhaps the ionized gas spiral in the Galactic center is also caused by a density wave. This possibility is supported by the fact that a one-armed spiral is the dominant instability in a disk with a nearly Keplerian rotation curve (Adams et al. 1989). However, both the ionized and the atomic gas in Sgr A West have densities which are much too small to support a gravitational instability, with Toomre $Q$ parameters (Binney \& Tremaine 1987) of $\sim 1000$ and $\sim 50$, respectively. The molecular gas in the CND could be gravitationally unstable if its mass is as large as concluded by Christopher et al. (2005) and MonteroCastaño et al. (2009). However, if the smaller mass derived from far-infrared dust emission (Etxaluze et al. 2011) is assumed, then even the CND should not support gravitational instabilities. The mass density of the stellar distribution is large enough to be gravitationally unstable if it is flattened into a disk, but the distribution of the older stars, which constitute the bulk of the stellar mass, is probably not highly flattened. We conclude that most likely the ionized spiral is not a result of a density wave caused by gravitational interactions within the disk. But a wave might be induced by another perturbing force, perhaps due to magnetic fields.

Alternatively, a perturbing force may not be necessary to organize the gas in Sgr A West into a spiral pattern. The reason that a one-armed spiral is the main instability in a potential dominated by a point mass is that orbits are approximately elliptical, with one focus at the center, so that if gas orbits are 


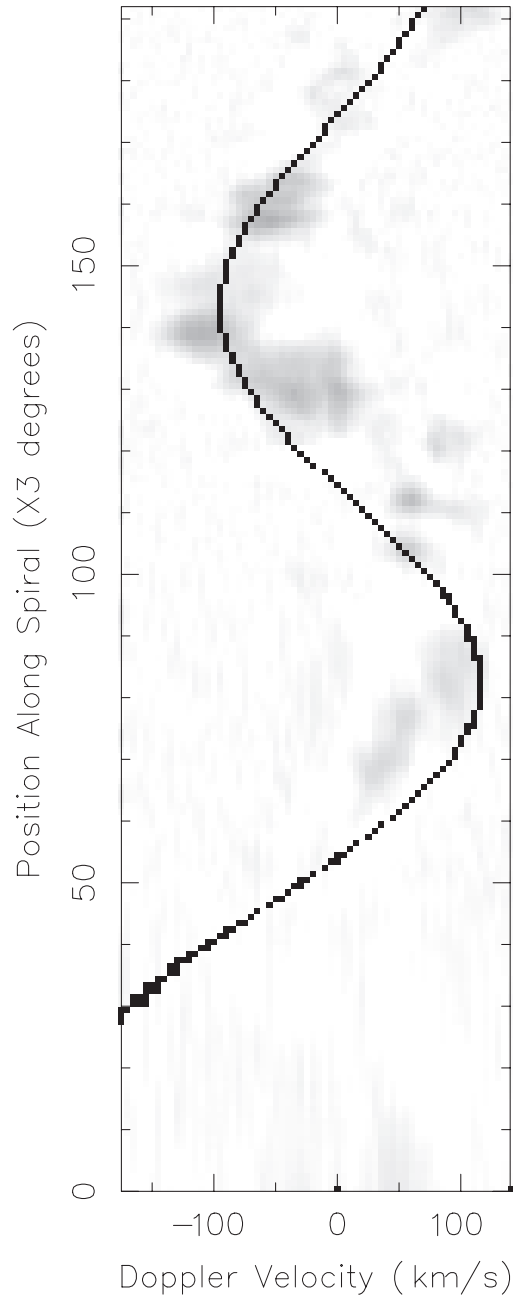

Figure 10. Position-velocity diagram of $\mathrm{HCN}(4-3)$ emission along the outside spiral in Figure $10\left(\phi=199^{\circ}\right)$; the vertical axis runs from $0^{\circ}$ to $576^{\circ}$ in $3^{\circ}$ steps; the horizontal axis runs from $-175 \mathrm{~km} \mathrm{~s}^{-1}$ to $+140 \mathrm{~km} \mathrm{~s}^{-1}$.

eccentric with orientations varying with radius, then they crowd along a one-armed spiral. In addition, the presence of distributed mass in the star cluster modifies the potential in such a way as to cause the orbits to precess, which could cause their orientations to vary with radius.

To investigate the possibility that gas orbits in the Galactic center gravitational potential naturally set up a spiral pattern, we ran a simulation of orbits in a mass distribution like that in Equation (1). We started the simulation with orbits along aligned ellipses, with all ellipses having one focus at Sgr $\mathrm{A}^{*}$ and a distance between the two foci varying as $a^{\gamma}$, with $\gamma=0-1$, and with the orbital plane uniformly populated with particles representing the gas. Each particle was allowed to orbit in the potential of a black hole plus a power-law stellar mass distribution, with no interactions between the particles. With this potential, orbits are well approximated with ellipses that precess in the retrograde direction. That is, the time from apocenter to apocenter is less than the time for a $360^{\circ}$ motion. For a mass distribution power law steeper than $r^{-1.5}$, inner orbits precess faster than outer, causing the orbits to crowd along a leading spiral; whereas for a shallower power law, outer orbits precess faster, causing a trailing spiral. The starting point of the simulation for an $r^{-0.5}$ stellar density distribution is shown in Figure 11(a), and the distribution of particles after $1.4 \times 10^{5} \mathrm{yr}$ is shown in Figure 11(b). The spiral persists for

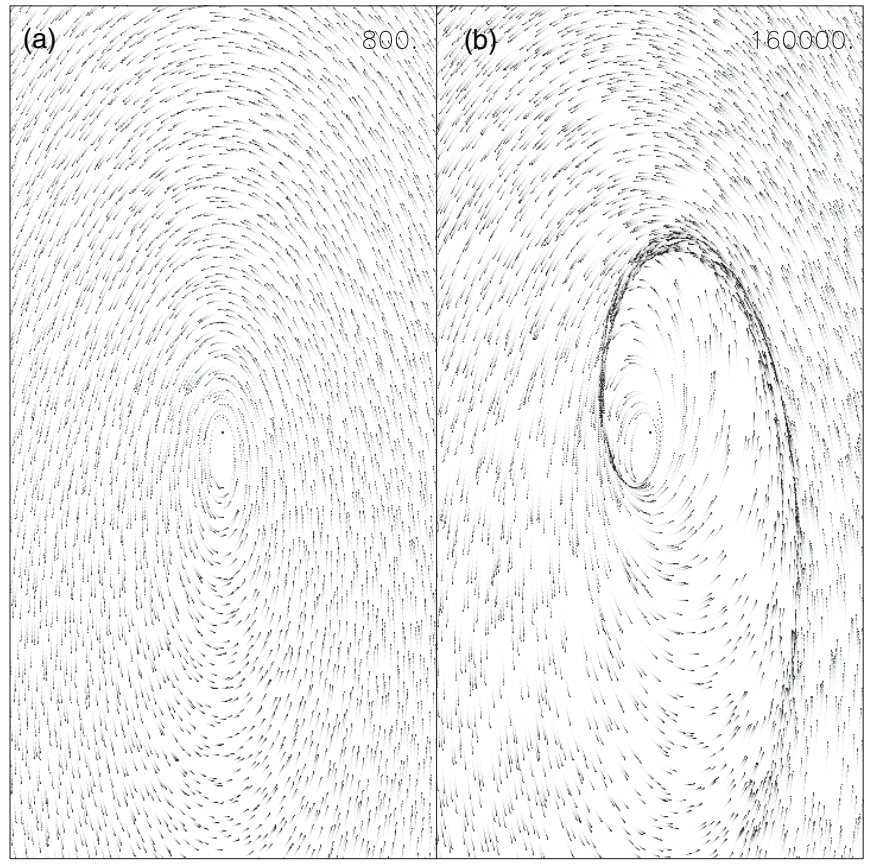

Figure 11. (a) Particle positions (with motions indicated with trails) just after the start of a simulation of orbits in the potential of a black hole and a flat stellar density distribution with $10^{5} M_{\odot}$ contained within $1 \mathrm{pc}$. The orbital plane is inclined by $65^{\circ}$ about a vertical axis. (b) Particle positions after $1.6 \times 10^{5} \mathrm{yr}$. The spiral wave is a result of the differential precession of the orbits; no interactions between the particles were included.

several times longer than it took to form since it wraps on a precession timescale, which is several $10^{5} \mathrm{yr}$, rather than the orbital timescale, which for the smallest orbits is several $10^{3} \mathrm{yr}$.

The spiral in the simulation strongly resembles that which we observe, in that it is quite open (not tightly wrapped) and approximately linear or Archimedean. In addition, the initial conditions of our simulation seem plausible as a situation that could result from the infall of a molecular cloud into the central region, and the relatively flat stellar density distribution required to produce a trailing spiral is consistent with recent observations (Buchholz et al. 2009; Do et al. 2009). We also note that the differential precession that causes the orbit crowding only occurs within $\sim 2 \mathrm{pc}$ of the center, consistent with the lack of a prominent spiral pattern in the molecular gas in the CND. Our main concern about this model is that in the time required for an orbit at $1.5 \mathrm{pc}$ to precess by $360^{\circ}$, gas at $0.1 \mathrm{pc}$ will have orbited around the center roughly 100 times. Whether gas could orbit this many times without being disrupted by stellar winds or other infalling clouds is unclear.

We also compared the spiral wave model to the kinematic distribution observed with the [Ne II] line by making a synthetic data cube with Doppler shifts calculated for orbits along a set of ellipses with orientations varying linearly with radius and eccentricities varying with radius to a power. The resulting orbits agree well with those in our simulation. The orbital velocities were calculated assuming constant angular momentum and energy along each ellipse. For our calculations, we used the effective potential:

$$
U=-G M_{\bullet} / r+\alpha r^{\beta}+l^{2} /\left(2 r^{2}\right),
$$

which corresponds to a stellar mass distribution varying as $r^{\beta-2}$. With this synthetic data cube, we could calculate the predicted Doppler shift at each point in the sky, allowing us to spectrally 

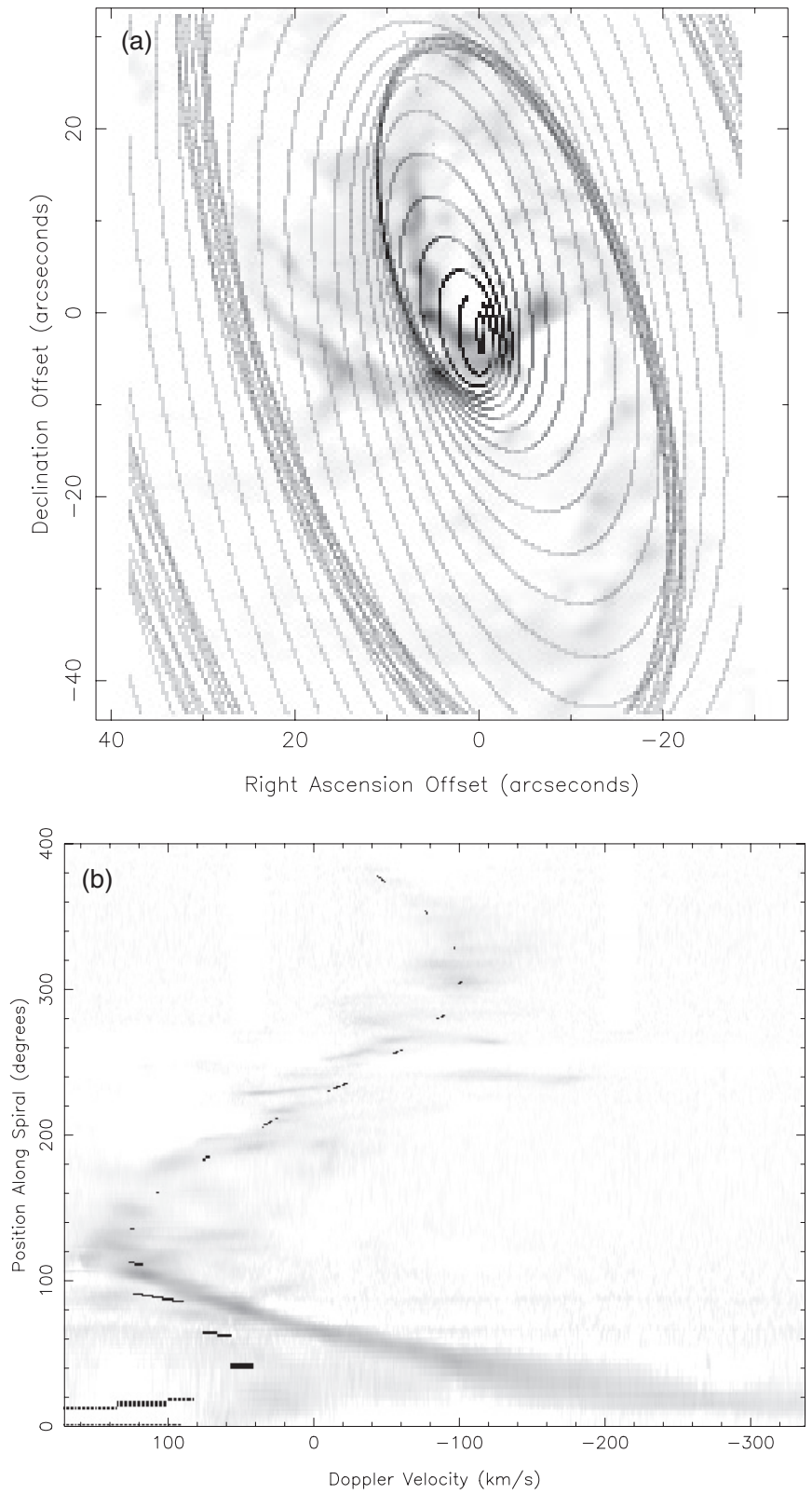

Figure 12. (a) The density wave model which gives the best fit to velocities in the entire data cube. (b) Position-velocity diagram extracted from along the [Ne II] spiral. The parameters for this fit are $\psi_{i}=118^{\circ}, d \psi / d a=214^{\circ} \mathrm{pc}^{-1}$, $e_{1}=0.3, b=-0.5, \alpha=500, \beta=1$, and $M_{\bullet}=4.5 \times 10^{6} M_{\odot}$. The spatial fit is poor as the model spiral is far outside the [Ne II] spiral. This affects the $P-V$ diagram in that the [Ne II] spiral crosses to the other side of the innermost ellipses, causing the spectral model to turn to the red.

shift and collapse our observed data cube as we did for our circular orbit model, and to search for parameters that give the best agreement between the model and the data. The relevant parameters are the orientation for $a=0\left(\psi_{i}\right)$, the rate of change of orientation $(d \psi / d a)$, and the rate of change in eccentricity (given by $e_{1}$, the eccentricity at $a=1 \mathrm{pc}$, and the power, $b$, such that $\left.e=e_{1}(a / 1 \mathrm{pc})^{b}\right)$, as well as the potential energy parameters, $M_{\bullet}, \alpha$, and $\beta$. We held the disk parameters constant at $l=66^{\circ}$ and $\Omega=15^{\circ}$. The best-fitting parameters were $\psi_{i}=118^{\circ}$, $d \psi / d a=214^{\circ} \mathrm{pc}^{-1}, e_{1}=0.3, b=-0.5, \alpha=500, \beta=1$, and $M_{\bullet}=4.5 \times 10^{6} M_{\odot}$. This corresponds to a stellar mass within $r=1 \mathrm{pc}$ of $1.16 \times 10^{5} M_{\odot}$. The spatial distribution of ellipses is shown plotted on the [Ne II] data in Figure 12(a), and
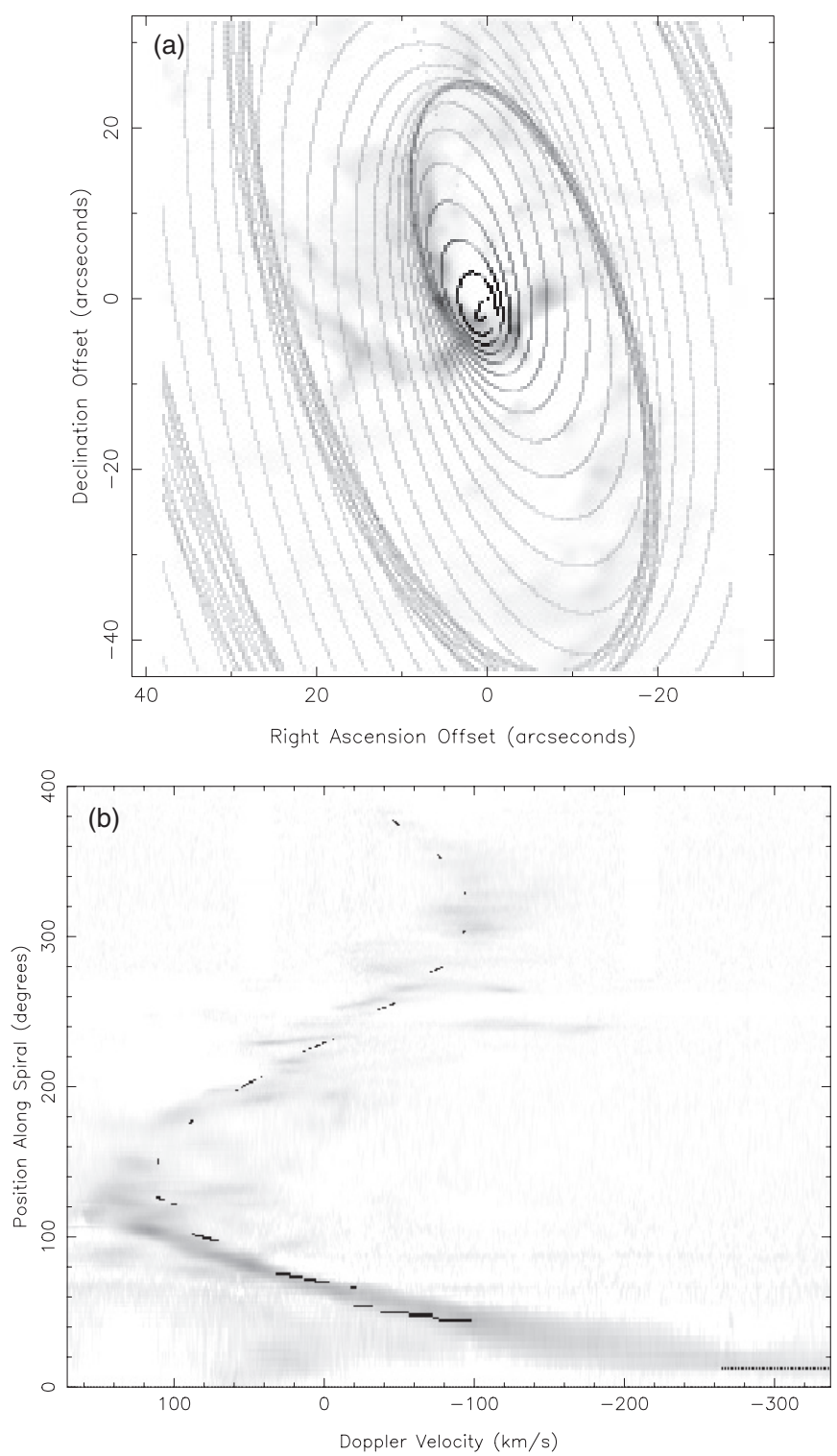

Figure 13. (a) The density wave model with the starting ellipse orientation adjusted to achieve the best fit to the spectra along the spiral. (b) Position-velocity diagram extracted from along the ionized gas spiral. Now, $\psi_{i}=155^{\circ}$. This shows a very good spectral fit, and the spatial fit shows a density peak just outside of the emission spiral.

a $P-V$ diagram extracted from along the northern arm and the western arc is shown in Figure 12(b). Note that the density wave spiral model lies significantly outside the [Ne II] spiral, which causes the model $P-V$ diagram in the northern arm to turn to the red. By adjusting the starting position, $\psi_{i}$, we can bring the model closer spatially to the data. With $\psi_{i}=175^{\circ}$, the density wave model lines up with the [Ne II] emission, and, perhaps as expected, the $P-V$ diagram looks like Figure 7(a) for motion along the spiral. With $\psi_{i}=155^{\circ}$, the density wave peak lies just outside the [Ne II] spiral (Figure 13), and the model $P-V$ pattern agrees well with the observations. We choose to manually adjust this parameter to specifically fit the observed spatial pattern. The original fit $\left(\psi_{i}=118^{\circ}\right)$ must have been affected by emission that is not part of the spiral pattern, resulting in a worse fit for the $P-V$ diagram. By adjusting $\psi_{i}$, we see a far superior fit to the spiral, while presumably causing a worse fit for the material elsewhere in the map. That the ionized gas peak would lie inside of the density peak (Figure 13) is sensible if the gas becomes ionized 
on entering the spiral or if ionizing radiation illuminates the inner edge of the spiral. It is important to note that we now get a mass distribution similar to that determined by stellar observers. This is a result of the fact that the orbit crowding is strongest just beyond apocenter where the particles are moving more slowly than the circular speeds and are moving slightly inward, which is consistent with the slight inward velocity derived for the circular orbit model.

To test this model more thoroughly would require a hydrodynamic, or perhaps a magneto-hydrodynamic simulation, which will have to be a project for the future.

\subsection{What We do not Explain}

One problem with a density wave model of the ionized spiral is explaining the high contrast seen, especially between the northern arm and the region just to its west, where the emission is a factor $\sim 100$ fainter. A possible explanation is that the gas is not just compressed in the spiral, but is also more highly ionized there. The gas could be largely neutral over most of its orbit, but become ionized as a result of passing through a shock or by interacting with the magnetic field when entering the northern arm and western arc. Although stellar ultraviolet radiation appears to be sufficient to account for the ionization of the gas in Sgr A West, the fact that the stars can only be observed in the infrared, making it difficult to determine their ultraviolet luminosities, leaves open the possibility of contribution by other sources of ionization. We can place an upper limit on the rate of conversion of kinetic energy into ionization in a shock by estimating the rate at which gas carries kinetic energy into the northern arm. If we estimate the mass of (predominantly neutral) gas in the inner $1.5 \mathrm{pc}$ to be $1000 M_{\odot}$, take a typical speed of $100 \mathrm{~km} \mathrm{~s}^{-1}$, and assume $1 / 4$ of its kinetic energy is dissipated once per orbit, or each time it passes through the spiral pattern, then we estimate a power of $2 \times 10^{4} L_{\odot}$. This is much less than the ionizing luminosity of $\sim 10^{6} L_{\odot}$ needed to maintain the ionization (Brown \& Liszt 1984). Apparently, shocks make at most a minor contribution to the ionization of the gas.

We also have not explained the orientation of the magnetic field that runs along the northern arm. We (perhaps naively) would have expected the field lines to be aligned with the gas motions, which we conclude run diagonal to the northern arm. But compression of a field in a spiral shock may instead cause the field to be aligned parallel to the shock. A magnetohydrodynamic simulation may be able to resolve this question.

Finally, we note that we have not proposed a model of the eastern arm and bar region, which account for about half of the ionic emission from Sgr A West. The elliptical orbit model of Zhao et al. (2009) fits the spatial distribution of these features rather well, but does not agree well with the observed [Ne II] kinematics. Neither of these features are fitted by circular motions of gas in a plane. As a speculative suggestion, we note the morphological similarity of the eastern arm, especially its northeastern loop (which is most prominent in the VLA free-free maps) to solar prominences. Perhaps magnetic fields very close to the center are strong enough to lift ionized gas out of the center along this feature. A measurement of the magnetic field in this region would be difficult due to the faintness of the dust emission there, but would be of interest.

\section{SUMMARY}

The [Ne II] observations strongly favor a model in which much of the ionized gas in Sgr A West orbits in a plane close to that of the CND, with nearly circular motions. The spatial pattern of the gas in this plane is well described by an approximately linear spiral, which includes the western arc and the northern arm. This model requires the gas orbits to cross the spiral, especially in the northern arm region.

We have considered several implications of and physical models to explain these conclusions. The most promising physical model involves a spiral density wave resulting from the precession of elliptical orbits in the potential of the central SMBH and star cluster. In addition to providing an explanation for the origin of the spiral, this model results in a best-fitting mass distribution in agreement with that derived from orbital motions of stars around the $\mathrm{SMBH}$, and with relatively flat stellar mass distribution in the inner parsec.

We thank Milos Milosavljevic for very helpful discussions and Thomas Greathouse for assistance with the observations. We also thank María Montero-Castaño for providing the HCN data. This work was supported by NSF grants AST-0607312 and AST-0708074.

\section{REFERENCES}

Adams, F. C., Ruden, S. P., \& Shu, F. H. 1989, ApJ, 347, 959

Aitken, D. K., Gezari, D., Smith, C. H., McCaughrean, M., \& Roche, P. F. 1991, ApJ, 380, 419

Aitken, D. K., Smith, C., Moore, T. J., \& Roche, P. F. 1998, MNRAS, 299, 743

Baganoff, F. K., Maeda, Y., Morris, M., et al. 2003, ApJ, 591, 891

Bahcall, J. N., \& Wolf, R. A. 1976, ApJ, 209, 214

Balick, B., \& Brown, R. L. 1974, ApJ, 194, 265

Bartko, H., Martins, F., Trippe, S., et al. 2010, ApJ, 708, 834

Becklin, E. E., Gatley, I., \& Werner, M. W. 1982, ApJ, 258, 135

Becklin, E. E., \& Neugebauer, G. 1968, ApJ, 151, 145

Binney, J., \& Tremaine, S. 1987, Galactic Dynamics (Princeton, NJ: Princeton Univ. Press), 747

Brown, R. L., \& Liszt, H. S. 1984, ARA\&A, 22, 223

Buchholz, R. M., Schödel, R., \& Eckart, A. 2009, A\&A, 499, 483

Christopher, M. H., Scoville, N. Z., Stolovy, S. R., \& Yun, M. S. 2005, ApJ, 622,346

Davidson, J. A., Werner, M. W., Wu, X., et al. 1992, ApJ, 387, 189

Do, T., Ghez, A. M., Morris, M. R., et al. 2009, ApJ, 703, 1323

Downes, D., \& Martin, A. H. M. 1971, Nature, 233, 112

Etxaluze, M., Smith, H. A., Tolls, V., Stark, A. A., \& González-Alfonso, E. 2011, AJ, 142, 134

Geballe, T. R., Rigaut, F., Roy, J.-R., \& Drain, B. T. 2004, ApJ, 602, 770

Genzel, R., Eisenhauer, F., \& Gillessen, S. 2010, Rev. Mod. Phys., 82, 3121

Genzel, R., Schödel, R., Ott, T., et al. 2003, ApJ, 594, 812

Gerhard, O. 2001, ApJ, 546, 39

Ghez, A. M., Salim, S., Weinberg, N., et al. 2008, ApJ, 689, 1044

Gillessen, S., Eisenhauer, F., Trippe, S., et al. 2009, ApJ, 692, 1075

Glasse, A., Aitken, D. K., \& Roche, P. F. 2003, Astron. Nachr. Suppl., 324,563

Güsten, R., Genzel, R., Wright, M., et al. 1987, ApJ, 318, 124

Herbst, T. M., Beckwith, S., Forrest, W. J., \& Pipher, J. L. 1993, AJ, 105, 956

Jackson, J. M., Geis, N., Genzel, R., et al. 1993, ApJ, 402, 173

Lacy, J. H., Achtermann, J. M., \& Serabyn, E. 1991, ApJ, 380, 71

Lacy, J. H., Richter, M. J., Greathouse, T. K., Jaffe, D. T., \& Zhu, Q. 2001, PASP, 114,153

Lacy, J. H., Townes, C. H., Geballe, T. R., \& Hollenbach, D. J. 1980, ApJ, 241,132

Levin, Y., \& Beloborodov, A. M. 2003, ApJ, 590, 33

Liszt, H. S. 2003, A\&A, 408, 1009

Lo, K. Y., \& Claussen, M. J. 1983, Nature, 306, 647

Merritt, D. 2010, ApJ, 718, 739

Mezger, P. G., Duschl, W. J., \& Zylka, R. 1996, A\&ARv, 7, 289

Mezger, P. G., Zylka, R., Salter, C. J., et al. 1989, A\&A, 209, 337

Montero-Castaño, M., Herrnstein, R. M., \& Ho, P. 2009, ApJ, 695, 1477

Morris, M., \& Serabyn, E. 1996, ARA\&A, 34, 645

Nityananda, R., \& Narayan, R. 1982, J. Astrophys. Astron., 3, 419

Oka, T., Nagai, M., Kamegai, K., \& Tanaka, K. 2011, ApJ, 732, 120

Paumard, T., Maillard, J. P., \& Morris, M. 2003, Astron. Nachr. Suppl., 324,605 
Paumard, T., Maillard, J. P., \& Morris, M. 2004, A\&A, 426, 81

Rieke, G. H., \& Rieke, M. J. 1988, ApJ, 330, 33

Roberts, D. A., \& Goss, W. M. 1993, ApJS, 86, 133

Sanders, R. H. 1998, MNRAS, 294, 35

Schödel, R., Eckart, A., Alexander, T., et al. 2007, A\&A, 469, 125

Serabyn, E., \& Lacy, J. H. 1985, ApJ, 293, 445
Serabyn, E., Lacy, J. H., \& Achtermann, J. M. 1991, ApJ, 378, 557

Serabyn, E., Lacy, J. H., Townes, C. H., \& Bharat, R. 1988, ApJ, 326, 171

Vollmer, B., \& Duschl, W. J. 2000, New Astron., 4, 581

Wollman, E. R., Geballe, T. R., Lacy, J. H., Townes, C. H., \& Rank, D. M. 1977, ApJ, 218, 103

Zhao, J.-H., Morris, M. R., Goss, W. M., \& An, T. 2009, ApJ, 699, 186 


\title{
ERRATUM: “IONIZED GAS IN THE GALACTIC CENTER: NEW OBSERVATIONS AND INTERPRETATION" (2012, ApJ, 755, 90)
}

\author{
Wesley T. Irons ${ }^{1,3}$, John H. LaCY ${ }^{1}$, and MatThew J. RichteR ${ }^{2}$ \\ ${ }^{1}$ Department of Astronomy, University of Texas, Austin, TX 78712, USA; lacy@astro.as.utexas.edu \\ ${ }^{2}$ Department of Physics, University of California, Davis, CA 95616, USA \\ Received 2013 May 15; published 2013 June 17
}

Online-only material: Supplemental data (FITS) file (tar.gz)

Due to an incorrect FITS keyword in the data associated with Figure 1, the velocity scale of the data cube was incorrect in the published version of this paper. The FITS file has now been corrected.

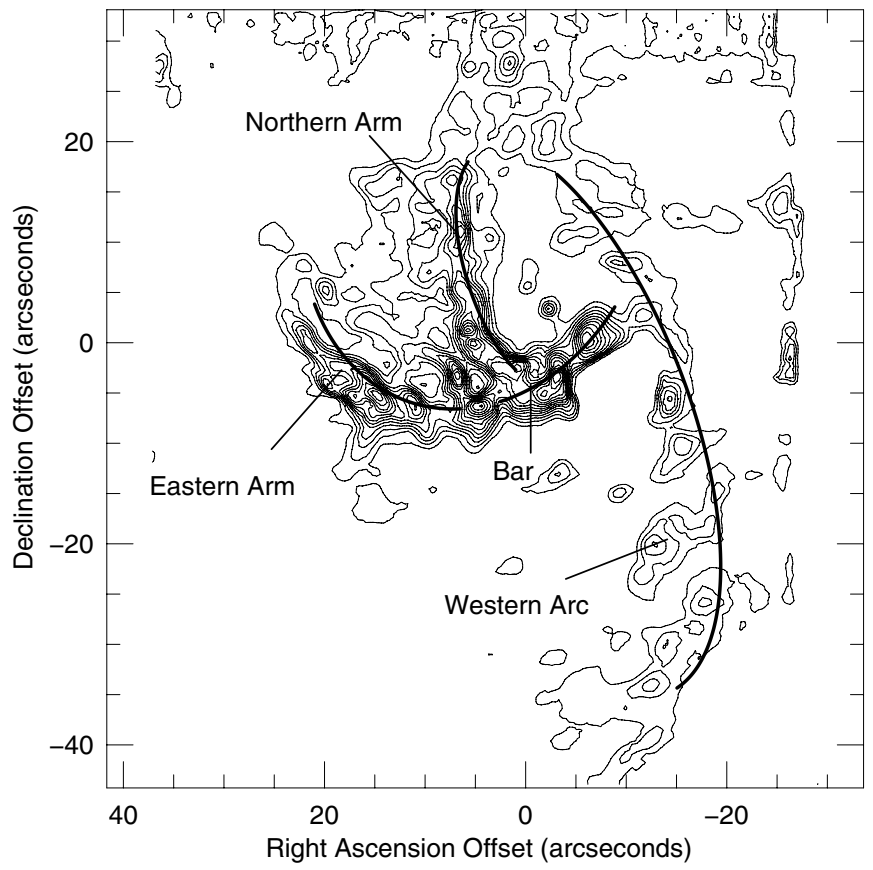

Figure 1. Contour plot of [Ne II] emission, with a nonlinear stretch, shown here with the various structures labeled. Offsets are from $\mathrm{Sgr} \mathrm{A}^{*}$ at $17^{\mathrm{h}} 45^{\mathrm{m}} 40^{\mathrm{s}} .04$ $29^{\circ} 00^{\prime} 28^{\prime \prime} 11(\mathrm{~J} 2000)$.

(Supplementary data (FITS images) of this figure are available in the online journal.)

We also note that the channels of the data cube are evenly spaced in frequency, not wavelength, so the velocity scale is strictly correct only at the center of the cube. The frequency scale in the FITS extension is correct, although it is not corrected for Earth's motion relative to the LSR. In addition, due to uncertainties in the telescope pointing and reduction of the data cube, coordinates may be uncertain by up to $1^{\prime \prime}$, and intensities are uncertain at the level of $\sim 20 \%$.

The first channel of the data cube has been replaced with the $12.8 \mu \mathrm{m}$ continuum, taken from channels between -430 and $-490 \mathrm{~km} \mathrm{~s}^{-1}$. The second channel is the [Ne II] line intensity, with continuum subtracted, integrated over channels between +344 and $-416 \mathrm{~km} \mathrm{~s}^{-1}$. It has units of erg $\left(\mathrm{s} \mathrm{cm}^{2} \mathrm{sr}\right)^{-1}$. The other maps have units of $\mathrm{erg}\left(\mathrm{s} \mathrm{cm}^{2} \mathrm{sr} \mathrm{cm}^{-1}\right)^{-1}$. The continuum has also been subtracted from the channel maps. The position of Sgr A* has been marked with the value of one in all maps.

\footnotetext{
3 Current address: Department of Physics and Astronomy, Johns Hopkins University, Baltimore, MD 20723, USA.
} 